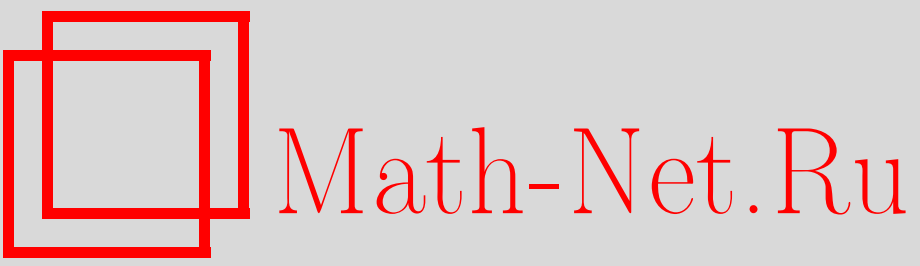

А. Ю. Попов, И. В. Тихонов, Экспоненциальные классы разрешимости в задаче теплопроводности с нелокальным условием среднего по времени, Матем. сб., 2005, том 196, номер 9, 71-102

DOI: https://doi.org/10.4213/sm1421

Использование Общероссийского математического портала Math-Net.Ru подразумевает, что вы прочитали и согласны с пользовательским соглашением

http: //www . mathnet.ru/rus/agreement

Параметры загрузки:

IP : 54.224 .187 .69

26 апреля 2023 г., 17:09:22 


\section{Экспоненциальные классы разрешимости в задаче теплопроводности с нелокальным условием среднего по времени}

При $x \in \mathbb{R}^{n}, 0 \leqslant t \leqslant T$ рассматривается нелокальная по времени задача для уравнения теплопроводности. Требуется найти функцию $u(x, t)$ из соотношений

$$
\frac{\partial u}{\partial t}=\Delta u, \quad \frac{1}{T} \int_{0}^{T} u(x, t) d t=\varphi(x) .
$$

Указана явная формула для решения. Изучен вопрос о ее применимости. Дано описание классов корректности. Основное предположение: при $|x| \rightarrow \infty$ решение $u(x, t)$ растет не быстрее $\exp (\sigma|x|)$ с показателем $\sigma<\sqrt{\pi / T}$.

Библиография: 17 названий.

Задачи теплопроводности с решениями, растушими при $|x| \rightarrow \infty$, привлекают внимание исследователей со времен классических работ [1]-[3]. Наиболее изученной является задача Коши, где наряду со стандартной техникой эффективно применяется теория обобщенных функций [4]. Результаты формулируются в терминах решений, растущих при $|x| \rightarrow \infty$ не быстрее, чем $\exp \left(\sigma|x|^{2}\right)$ (или в каких-то близких по типу классах).

В настоящей работе нас интересует другая ситуация, когда вместо условия Коши задан интеграл по времени $t$ на некотором отрезке $[0, T]$. “Абстрактная" теория единственности для подобных задач была разработана в [5]. На ее основе в [6], [7] получено полное описание классов единственности для уравнения теплопроводности в $\mathbb{R}^{n}$. Результаты сформулированы в терминах решений, растущих при $|x| \rightarrow \infty$ не быстрее $\exp (\sigma|x|)$, причем граница между единственностью и неединственностью определяется значением $\sigma=\sqrt{\pi / T}$. Вопрос о разрешимости в [6], [7] не рассматривался. В настоящей работе мы устраним этот пробел, указав необходимые и достаточные условия разрешимости. Кроме того, получим явную формулу для решений и дадим ее обоснование. В работе используется та же шкала роста $\exp (\sigma|x|)$.

Заметим, что в [6], [7] вводилась еще одна, более тонкая шкала $|x|^{a} \exp (\sigma|x|)$ с границей $a=-(n-1) / 2, \sigma=\sqrt{\pi / T}$ между единственностью и неединственностью. Однако исследование разрешимости в такой “тонкой” шкале становится довольно громоздким. Поэтому на данном этапе мы ограничились простой шкалой $\exp (\sigma|x|)$.

Основной результат представлен в теореме 3 из $\S 6$. Более простые предварительные версии даны в теоремах 1 и 2 из $\S \S 4$ и 6 соответственно; дополнительные уточнения сделаны в теоремах 5,6 из $§ 10$. Центральное соотношение - формула (11) для начального состояния $u_{0}(x)$; ее обобщение - формула $(21)$. Здесь 
фигурирует “функция Грина” $g_{T}(x)$, которая подробно изучается в $\S 7$. В частности, доказывается важная теорема 4 о разложении $g_{T}(x)$ в сходящийся ряд по функциям Ханкеля, откуда выводятся необходимые оценки $g_{T}(x)$ на бесконечности. Остальное содержание работы видно из названий параграфов. Рассмотрение ведется классическими средствами, теория обобщенных функций не используется.

\section{§1. Постановка задачи}

Фиксируем натуральное число $n \geqslant 1$ и вешественное $T>0$. Рассмотрим задачу о нахождении функции $u(x, t), x=\left(x_{1}, \ldots, x_{n}\right) \in \mathbb{R}^{n}, 0 \leqslant t \leqslant T$, из соотношений

$$
\begin{array}{cl}
\frac{\partial u}{\partial t}=\Delta u, & \Delta \equiv \frac{\partial^{2}}{\partial x_{1}^{2}}+\cdots+\frac{\partial^{2}}{\partial x_{n}^{2}}, \quad x \in \mathbb{R}^{n}, \quad 0<t \leqslant T, \\
\frac{1}{T} \int_{0}^{T} u(x, t) d t=\varphi(x), \quad x \in \mathbb{R}^{n} .
\end{array}
$$

Функция $\varphi \in C\left(\mathbb{R}^{n}\right)$ предполагается заданной. Множитель $1 / T$ в формуле $(2)$ взят для удобства - он позволяет считать $\varphi(x)$ в точности средним значением "температуры" $u(x, t)$ по времени $t$. Очевидно, что (2) эквивалентно условию

$$
\int_{0}^{T} u(x, t) d t=\psi(x), \quad x \in \mathbb{R}^{n}
$$

где $\psi(x)=T \varphi(x)$. Последующие результаты сразу переносятся с задачи $(1),(2)$ на $(1),(3)$. Поэтому будем рассматривать только задачу $(1),(2)$. Через $\psi(x)$ будем часто обозначать интеграл, вычисленный для $u(x, t)$ по формуле $(3)$.

Решением задачи (1), (2) назовем функцию $u(x, t)$ из класса

$$
C^{2,1}\left(\mathbb{R}^{n} \times(0, T]\right) \cap C\left(\mathbb{R}^{n} \times[0, T]\right),
$$

удовлетворяюшую уравнению (1) в классическом смысле при $x \in \mathbb{R}^{n}, 0<t \leqslant T$ и обращаюшую соотношение (2) в верное тождество всюду в $\mathbb{R}^{n}$.

Недавно в работе [7] авторами установлено, что решение задачи (1), (2) будет единственным в классе функций $u(x, t)$, растущих при $|x| \rightarrow \infty$ не быстрее $\exp (\sigma|x|)$ с показателем $\sigma<\sqrt{\pi / T}$. При $\sigma=\sqrt{\pi / T}$ единственность решения уже нарушается (см. теоремы 1, 3 в [7]; см. также [6]).

В настоящей работе будет доказано, что в этих же экспоненциальных классах единственности (c $\sigma<\sqrt{\pi / T})$ задача $(1)$, (2) будет заведомо разрешимой при некоторых естественных ограничениях на функцию $\varphi(x)$ и неразрешимой, если такие ограничения не выполняются (см. теоремы 1-3 ниже).

Все интересующие нас решения представимы интегральной формулой Пуассона

$$
\left.u(x, t)\right|_{0<t \leqslant T}=\frac{1}{(4 \pi t)^{n / 2}} \int_{\mathbb{R}^{n}} \exp \left(-\frac{|x-y|^{2}}{4 t}\right) u_{0}(y) d y, \quad u(x, 0)=u_{0}(x) .
$$

Начальное состояние $u_{0} \in C\left(\mathbb{R}^{n}\right)$ будет всегда выбираться так, чтобы гарантировать принадлежность функции $u(x, t)$, определенной по формуле $(4)$, классу 
$C^{2,1}\left(\mathbb{R}^{n} \times(0, T]\right) \cap C\left(\mathbb{R}^{n} \times[0, T]\right)$. Поэтому задачу (1), (2) можно трактовать как задачу о нахождении неизвестного начального состояния $u_{0}(x)$, при котором функция $u(x, t)$ из формулы (4) обрашает соотношение (2) в верное тождество.

Начнем изучение задачи $(1),(2)$ с вывода явной формулы для $u_{0}(x)$ при помощи преобразования Фурье. На этом этапе рассуждения носят "формальный” характер.

\section{§ 2. Формальное преобразование Фурье}

Пусть

$$
U(\xi, t)=\int_{\mathbb{R}^{n}} u(x, t) \exp (-i \xi x) d x, \quad \Phi(\xi)=\int_{\mathbb{R}^{n}} \varphi(x) \exp (-i \xi x) d x
$$

- формальные преобразования Фурье по переменной $x \in \mathbb{R}^{n}$. Здесь, как обычно, $\xi=\left(\xi_{1}, \ldots, \xi_{n}\right) \in \mathbb{R}^{n}, \xi x=\xi_{1} x_{1}+\cdots+\xi_{n} x_{n}, i^{2}=-1$.

Соотношения (1), (2) переходят в следующие:

$$
\frac{\partial U(\xi, t)}{\partial t}=-|\xi|^{2} U(\xi, t), \quad \frac{1}{T} \int_{0}^{T} U(\xi, t) d t=\Phi(\xi) .
$$

Из дифференциального уравнения следует, что $U(\xi, t)=\exp \left(-|\xi|^{2} t\right) U_{0}(\xi)$. Подставим эту функцию в интеграл, вычислим его и выразим $U_{0}(\xi)$. Получим

$$
U_{0}(\xi)=\frac{T|\xi|^{2}}{1-\exp \left(-T|\xi|^{2}\right)} \Phi(\xi) \equiv P_{T}(\xi) \Phi(\xi) .
$$

Функция

$$
P_{T}(\xi) \equiv \frac{T|\xi|^{2}}{1-\exp \left(-T|\xi|^{2}\right)}=\frac{T|\xi|^{2} \exp \left(T|\xi|^{2}\right)}{\exp \left(T|\xi|^{2}\right)-1}
$$

при $|\xi| \rightarrow \infty$ ведет себя как $T|\xi|^{2}$, и, значит, ей не соответствует никакое преобразование Фурье от классического объекта. Выделим в (7) слагаемое $T|\xi|^{2}$ и запишем

$$
U_{0}(\xi)=\frac{T|\xi|^{2}}{\exp \left(T|\xi|^{2}\right)-1} \Phi(\xi)+T|\xi|^{2} \Phi(\xi) \equiv G_{T}(\xi) \Phi(\xi)+T|\xi|^{2} \Phi(\xi) .
$$

Здесь

$$
G_{T}(\xi) \equiv \frac{T|\xi|^{2}}{\exp \left(T|\xi|^{2}\right)-1}, \quad \xi \in \mathbb{R}^{n} .
$$

Обозначим $Q(s) \equiv s(\exp (s)-1)^{-1}, s \in \mathbb{R}$, с обычным соглашением $Q(0)=1$. Функция $Q(s)$ бесконечно дифференцируема по $s \in \mathbb{R}$. Она сама и все ее производные стремятся к нулю при $s \rightarrow+\infty$ быстрее любой степени $s$. Следовательно, функция $G_{T}(\xi)=Q\left(T|\xi|^{2}\right)$ принадлежит пространству Шварца $S\left(\mathbb{R}^{n}\right)$ быстро убьваюших бесконечно дифференцируемых функций. Обратное преобразование Фурье корректно определено формулой

$$
g_{T}(x)=\frac{1}{(2 \pi)^{n}} \int_{\mathbb{R}^{n}} \frac{T|\xi|^{2}}{\exp \left(T|\xi|^{2}\right)-1} \exp (i \xi x) d \xi, \quad x \in \mathbb{R}^{n},
$$


и также принадлежит пространству $S\left(\mathbb{R}^{n}\right)$. Интеграл в (10) быстро сходится, функция $g_{T}(x)$ является целой ${ }^{1}$ по переменной $x \in \mathbb{R}^{n}$. Асимптотические свойства $g_{T}(x)$ при $|x| \rightarrow \infty$ подробно исследуются в $\S 7$ (см. также лемму 2 в $\left.\S 3\right)$. Из (10) следует, что $g_{T}(x)=T^{-n / 2} g_{1}\left(T^{-1 / 2} x\right)$, но мы будем рассматривать $g_{T}(x)$ независимо, не используя выражение через $g_{1}(x)$.

Применим в (8) обратное преобразование Фурье. Учитывая теорему о свертке и то, что $|\xi|^{2} \Phi(\xi)$ переходит в $(-\Delta \varphi(x))$, получаем выражение для начального состояния $u_{0}(x)$ :

$$
u_{0}(x)=\int_{\mathbb{R}^{n}} g_{T}(x-y) \varphi(y) d y-T \Delta \varphi(x), \quad x \in \mathbb{R}^{n},
$$

с функцией $g_{T}(x)$ из формулы $(10)$. Будем считать $g_{T}(x)$ функцией Грина для рассматриваемой задачи $(1),(2)$. Особая связь $g_{T}(x)$ с данной задачей окончательно раскрывается далее (см. замечание 2 после формулировки леммы 8 в $\S 8$ ).

В случае, когда $\varphi(x)$ и $\Phi(\xi)$ быстро убывают на бесконечности, можно не разбивать (6) на два слагаемых, а записать непосредственно:

$$
u_{0}(x)=\frac{1}{(2 \pi)^{n}} \int_{\mathbb{R}^{n}} P_{T}(\xi) \Phi(\xi) \exp (i \xi x) d \xi, \quad x \in \mathbb{R}^{n}
$$

с функцией $P_{T}(\xi)$ из формулы $(7), P_{T}(\xi)=G_{T}(\xi)+T|\xi|^{2}$. Соотношение $(12)$ определяет псевдодифференииальный оператор (ПДО) от функции $\varphi(x)$, причем $P_{T}(\xi)$ является символом данного ПДО. Поскольку $P_{T}(\xi)$ не зависит от $x$, имеем ПДО с постоянными коэффициентами (см. [8; гл. I, § 1, п. 1.8]).

Естественно ожидать, что функция $u(x, t)$, построенная по формуле Пуассона (4) для указанного начального состояния $u_{0}(x)$, будет решением задачи $(1),(2)$. Этот факт нетрудно обосновать при помощи классического ( "неформального") преобразования Фурье в пространствах типа $S\left(\mathbb{R}^{n}\right)$ или $L^{2}\left(\mathbb{R}^{n}\right)$, где формулы (5) и (12) приобретают содержательный смысл. Но мы пойдем другим путем ${ }^{2}$. Покажем, что правило (11) является более универсальным - оно применимо в пространствах функций экспоненциального роста при условии, что показатель роста меньше, чем $\sqrt{\pi / T}$.

\section{§3. Экспоненциальные классы функций}

Пусть $\sigma$ - фиксированное вещественное число $(-\infty<\sigma<+\infty)$. Обозначим через $\mathscr{E}_{\sigma}\left(\mathbb{R}^{n} \times[0, T]\right)$ класс функций $u(x, t)$, заданных на $\mathbb{R}^{n} \times[0, T]$, для которых справедлива оценка

$$
|u(x, t)| \leqslant M \exp (\sigma|x|), \quad x \in \mathbb{R}^{n}, \quad 0 \leqslant t \leqslant T,
$$

\footnotetext{
1Это утверждение допускает двойную трактовку. Во-первых, $g_{T}(x)$ аналитически продолжается в $\mathbb{C}^{n}$ при замене в $(10)$ переменной $x=\left(x_{1}, \ldots, x_{n}\right)$ на $z=\left(z_{1}, \ldots, z_{n}\right)$. Полученный интеграл сходится при всех $z \in \mathbb{C}^{n}$. Во-вторых, из формулы (51) (см. далее в работе) следует, что $g_{T}(x)$ является целой функцией от $|x|^{2}=x_{1}^{2}+\cdots+x_{n}^{2}$.

${ }^{2} \mathrm{~B}$ нашем исследовании теория ПДО никак не используется. Было бы интересно узнать, даст ли ее применение что-то принципиально новое для рассматриваемой задачи (1), (2).
} 
с какой-то константой $M>0$, зависящей от функции $u$. Аналогично через $\mathscr{E}_{\sigma}\left(\mathbb{R}^{n}\right)$ обозначим класс функций $f(x)$, заданных на $\mathbb{R}^{n}$, для которых

$$
|f(x)| \leqslant M \exp (\sigma|x|), \quad x \in \mathbb{R}^{n}
$$

с константой $M>0$, зависящей от функции $f$.

В дальнейшем без оговорок используется естественная связь между оценками вида (13) и (14). Например, если непрерывная функция $u(x, t)$ принадлежит классу $\mathscr{E}_{\sigma}\left(\mathbb{R}^{n} \times[0, T]\right)$, то ее интеграл (3) принадлежит $\mathscr{E}_{\sigma}\left(\mathbb{R}^{n}\right) ;$ кроме того, $u(\cdot, t) \in$ $\mathscr{E}_{\sigma}\left(\mathbb{R}^{n}\right)$ при каждом $t \in[0, T]$. Из формулы Пуассона (4) без труда выводится следующий “обратный” факт.

Лемма 1. Пусть функиия и $(x, t)$ выражсается на $\mathbb{R}^{n} \times[0, T]$ по формуле (4) с непрерывной функиией $u_{0}(x)$ из класса $\mathscr{E}_{\sigma}\left(\mathbb{R}^{n}\right)$. Тогда $u(x, t)$ принадлежит $\mathscr{E}_{\sigma}\left(\mathbb{R}^{n} \times[0, T]\right)$ с тем жее значением $\sigma \in \mathbb{R}$.

ДокАЗАТЕЛЬСтво. При $\sigma=0$ утверждение леммы общеизвестно. Пусть $\sigma \neq 0$ и $\left|u_{0}(x)\right| \leqslant M \exp (\sigma|x|)$ с константой $M>0$. Оценивая $u(x, t)$ при $t>0$, имеем

$$
\begin{aligned}
|u(x, t)| & \leqslant \frac{1}{(4 \pi t)^{n / 2}} \int_{\mathbb{R}^{n}} e^{-|x-y|^{2} /(4 t)} M e^{\sigma|y|} d y \\
& =\frac{M e^{\sigma|x|}}{(4 \pi t)^{n / 2}} \int_{\mathbb{R}^{n}} e^{-|y|^{2} /(4 t)} e^{\sigma(|x-y|-|x|)} d y
\end{aligned}
$$

Из неравенства треугольника следует, что $\sigma(|x-y|-|x|) \leqslant \sigma|y|$ при $\sigma>0$ и $\sigma(|x-y|-|x|) \leqslant-\sigma|y|$ при $\sigma<0$. Таким образом,

$$
|u(x, t)| \leqslant \frac{M e^{\sigma|x|}}{(4 \pi t)^{n / 2}} \int_{\mathbb{R}^{n}} e^{-|y|^{2} /(4 t)} e^{|\sigma| \cdot|y|} d y .
$$

Учитывая, что $|y| \leqslant\left|y_{1}\right|+\cdots+\left|y_{n}\right|$, получаем

$$
\begin{aligned}
|u(x, t)| & \leqslant M e^{\sigma|x|}\left(\frac{1}{\sqrt{4 \pi t}} \int_{-\infty}^{\infty} e^{-s^{2} /(4 t)} e^{|\sigma| \cdot|s|} d s\right)^{n} \\
& \leqslant M e^{\sigma|x|}\left(\frac{2}{\sqrt{4 \pi t}} \int_{-\infty}^{\infty} e^{-s^{2} /(4 t)} e^{|\sigma| s} d s\right)^{n}
\end{aligned}
$$

Последнее выражение в скобках $(\ldots)^{n}$ равно $2 \exp \left(\sigma^{2} t\right)$. Заменяя его максимальным значением на промежутке $(0, T]$, заключаем, что

$$
|u(x, t)| \leqslant M M^{*} \exp (\sigma|x|), \quad x \in \mathbb{R}^{n}, \quad 0 \leqslant t \leqslant T,
$$

где $M^{*}=2^{n} \exp \left(n \sigma^{2} T\right)$. Лемма доказана.

Для наших целей важно знать асимптотическое поведение при $|x| \rightarrow \infty$ функции $g_{T}(x)$ из формулы (10). Элементарные рассуждения показывают, что $\left|g_{T}(x)\right|$ не может стремиться к нулю слиштом быстро. 
Лемма 2. Функция $g_{T}(x)$, определенная формулой (10), не принадлежит $\mathscr{E}_{\sigma}\left(\mathbb{R}^{n}\right)$ ни при каком $\sigma<-\sqrt{\pi / T}$.

ДокаЗАтЕЛьство. Допустим, что $\left|g_{T}(x)\right| \leqslant M \exp (\sigma|x|)$ при $x \in \mathbb{R}^{n}$ с некоторым $\sigma<0$. Покажем, что тогда $\sigma \geqslant-\sqrt{\pi / T}$. Функция $G_{T}(\xi)$ из формулы (9) является преобразованием Фурье для $g_{T}(x)$. Выберем аргумент $\xi \in \mathbb{R}^{n}$ "одномерньгм" вида $(s, 0, \ldots, 0), s \in \mathbb{R}$, и запишем

$$
\frac{T s^{2}}{\exp \left(T s^{2}\right)-1}=\int_{\mathbb{R}^{n}} g_{T}(x) \exp \left(-i s x_{1}\right) d x .
$$

Аналитически продолжим последнее равенство в комплексную плоскость, заменив $s$ на $z=s+i \tau \mathrm{c}|\tau| \equiv|\operatorname{Im} z|<-\sigma$. Эта процедура законна - в силу оценки для $\left|g_{T}(x)\right|$ интеграл сходится равномерно на любом компакте в указанной полосе и там

$$
\left|\frac{T z^{2}}{\exp \left(T z^{2}\right)-1}\right| \leqslant \int_{\mathbb{R}^{n}} M e^{\sigma|x|} e^{\tau x_{1}} d x \leqslant M \int_{\mathbb{R}^{n}} e^{(\sigma+|\tau|)|x|} d x<\infty
$$

ибо $\sigma+|\tau|<0$. Но $z_{1} \equiv(1+i) \sqrt{\pi / T}-$ полюс функции $T z^{2}\left(\exp \left(T z^{2}\right)-1\right)^{-1}$ и $\operatorname{Im} z_{1}=\sqrt{\pi / T}$. Поэтому $\sqrt{\pi / T} \geqslant-\sigma$ и $\sigma \geqslant-\sqrt{\pi / T}$. Лемма доказана.

Далее, в $\S 7$, мы покажем, что численная граница $(-\sqrt{\pi / T})$, найденная в лемме 2 , является точной и $g_{T} \in \mathscr{E}_{\sigma}\left(\mathbb{R}^{n}\right)$ при $\sigma=-\sqrt{\pi / T}$. Здесь доказательство намного сложнее, но в конечном счете оно также сводится к тому, что $\sqrt{\pi / T}$ есть мнимая часть “первого” полюса $z_{1}=(1+i) \sqrt{\pi / T}$ функции $T z^{2}\left(\exp \left(T z^{2}\right)-1\right)^{-1}$.

\section{§4. Предварительный результат о разрешимости и необходимость в его обобщении}

Классы $\mathscr{E}_{\sigma}\left(\mathbb{R}^{n} \times[0, T]\right)$ тесно связаны с изучаемой задачей $(1),(2)$. Из результатов [7] следует, что задача (1), (2) не может иметь двух различных решений в $\mathscr{E}_{\sigma}\left(\mathbb{R}^{n} \times[0, T]\right)$ при $\sigma<\sqrt{\pi / T}$. И наоборот, при $\sigma \geqslant \sqrt{\pi / T}$ если решение сушествует, то оно заведомо неединственно. Таким образом, в классах $\mathscr{E}_{\sigma}\left(\mathbb{R}^{n} \times[0, T]\right)$ с $\sigma \geqslant \sqrt{\pi / T}$ интегральное условие (2) не определяет однозначно решение уравнения теплопроводности - задача $(1),(2)$ оказывается некорректной. Поэтому будем изучать (1), (2) только в классах $\mathscr{E}_{\sigma}\left(\mathbb{R}^{n} \times[0, T]\right)$ с $\sigma<\sqrt{\pi / T}$. Кроме того, пусть пока $\sigma>-\sqrt{\pi / T}$. Это ограничение подсказывается уже леммой 2. Там утверждается, что функция $g_{T}(x)$ стремится к нулю “не слишком быстро". Поэтому при свертке $g_{T}(x)$ по формуле $(11)$ с функцией $\varphi(x)$, стремящейся к нулю быстрее, чем $g_{T}(x)$, трудно гарантировать, что полученное начальное состояние $u_{0}(x)$ будет так же быстро (как $\varphi(x))$ стремиться к нулю. Короче говоря, классы экспоненциально убывающих функций с $\sigma \leqslant-\sqrt{\pi / T}$ имеют свою специфику (подробности см. в теореме 3 и в $\S 9)$.

Случай $|\sigma|<\sqrt{\pi / T}$ является "регулярным". Удобная и простая форма теоремы о разрешимости выглядит так. 
TеОрема 1. Пусть $\varphi \in C^{2}\left(\mathbb{R}^{n}\right) \cap \mathscr{E}_{\sigma}\left(\mathbb{R}^{n}\right)$ u $\Delta \varphi \in \mathscr{E}_{\sigma}\left(\mathbb{R}^{n}\right)$ при некотором фиксированном $\sigma,|\sigma|<\sqrt{\pi / T}$. Тогда задача $(1),(2)$ имеет решение $u(x, t)$, принадлежсащее $\mathscr{E}_{\sigma}\left(\mathbb{R}^{n} \times[0, T]\right)$. Это решение выражсается по формулам (4), (11), причем функиия $g_{T}(x)$ в формуле (11) определяется формулой (10). Других решений у задачи (1), (2) в указанном классе $\mathscr{E}_{\sigma}\left(\mathbb{R}^{n} \times[0, T]\right)$ нет.

Теорема 1 непосредственно следует из более общих теорем 2,3 (см. §6). Необходимость в обобщении объясняется тем, что даже в простом классе $\mathscr{E}_{0}\left(\mathbb{R}^{n} \times[0, T]\right)$ теорема 1 не охватывает все возможные ситуации. При $n \geqslant 2$ для произвольного ограниченного решения $u \in C^{2,1}\left(\mathbb{R}^{n} \times(0, T]\right) \cap C\left(\mathbb{R}^{n} \times[0, T]\right)$ уравнения теплопроводности (1) интеграл в условии (2) может непринадлежать $C^{2}\left(\mathbb{R}^{n}\right)$ и оператор Лапласа от функции $\varphi(x)$ будет не определен. Такие решения нельзя восстановить при помоши теоремы 1. Указанньй момент важен для дальнейшего, поэтому поясним его подробнее.

ЛЕмма 3. При любом $n \geqslant 2$ существует функция $и(x, t)$, определенная на $\mathbb{R}^{n} \times[0, T]$ по формуле Пуассона (4) с непрерывной ограниченной функиией $u_{0}(x)$, такая, что интеграл $\psi(x)$ из формуль (3) не принадлежит $C^{2}\left(\mathbb{R}^{n}\right)$.

ДокАЗАТЕЛЬСтво. Напомним, что оператор $\Delta$ является замыкаемым (но не замкнутым!) в $C\left(\mathbb{R}^{n}\right)$. Это свойство (замыкаемость) можно выразить так. Пусть $\varepsilon>0$ - малый параметр, $w_{\varepsilon} \in C^{2}\left(\mathbb{R}^{n}\right)$ и $\Delta w_{\varepsilon}(x)=f_{\varepsilon}(x)$ в $\mathbb{R}^{n}$. Если $w_{\varepsilon}(x) \rightarrow 0$ и $f_{\varepsilon}(x) \rightarrow f_{0}(x)$ при $\varepsilon \rightarrow 0$ равномерно на любом компакте в $\mathbb{R}^{n}$, то $f_{0}(x) \equiv 0$ всюду в $\mathbb{R}^{n}$ (см., например, [7; лемма 1]; отметим, что замыкание для $\Delta$ - расширенный оператор Лапласа $\Delta^{*}$ - рассматривается в следующем $\S 5$ настоящей статьи).

Выберем теперь функцию $u_{0}(x)$, непрерывную и ограниченную в $\mathbb{R}^{n}, n \geqslant 2$, так, чтобы уравнение $\Delta v(x)=u_{0}(x)$ не имело решений $v(x)$ класса $C^{2}$ в шаре $|x|<r$ с некоторым $r>0$ (см. [9; гл. IV, §3, пример с задачей (20), (22)]). Рассмотрим решение $u(x, t)$ уравнения теплопроводности $(1)$, полученное для $u_{0}(x)$ по формуле Пуассона (4), и обозначим как $\psi(x)$ интеграл $(3)$ от $u(x, t)$. Покажем, что $\psi(x)$ не может принадлежать $C^{2}\left(\mathbb{R}^{n}\right)$.

Допустим, что это не так и $\psi \in C^{2}\left(\mathbb{R}^{n}\right)$. Из формулы Пуассона следует, что $u \in C^{2,1}\left(\mathbb{R}^{n} \times(0, T]\right)$. Поэтому на любом отрезке $[\varepsilon, \tau] \subset(0, T]$ (в том числе на $[\varepsilon, T])$ интеграл по $t$ от $u(x, t)$ принадлежит $C^{2}\left(\mathbb{R}^{n}\right)$ и

$$
\Delta \int_{\varepsilon}^{\tau} u(x, t) d t=\int_{\varepsilon}^{\tau} \Delta u(x, t) d t=\int_{\varepsilon}^{\tau} \frac{\partial u(x, t)}{\partial t} d t=u(x, \tau)-u(x, \varepsilon) .
$$

Но тогда интеграл

$$
\int_{0}^{\varepsilon} u(x, t) d t=\int_{0}^{T} u(x, t) d t-\int_{\varepsilon}^{T} u(x, t) d t=\psi(x)-\int_{\varepsilon}^{T} u(x, t) d t
$$

также принадлежит $C^{2}\left(\mathbb{R}^{n}\right)$ и

$$
\Delta \int_{0}^{\varepsilon} u(x, t) d t=\Delta \psi(x)-u(x, T)+u(x, \varepsilon) .
$$


Ясно, что

$$
\int_{0}^{\varepsilon} u(x, t) d t \rightarrow 0, \quad \Delta \psi(x)-u(x, T)+u(x, \varepsilon) \rightarrow \Delta \psi(x)-u(x, T)+u_{0}(x)
$$

при $\varepsilon \rightarrow 0$ равномерно на любом компакте в $\mathbb{R}^{n}$. Используя в (16) замькаемость $\Delta$, получаем, что $\Delta \psi(x)-u(x, T)+u_{0}(x) \equiv 0$ в $\mathbb{R}^{n}$. Таким образом, уравнение $\Delta v(x)=u(x, T)-u_{0}(x)$ имеет классическое решение $v=\psi(x)$, определенное всюду в $\mathbb{R}^{n}$ и, в частности, в шаре $|x|<r$. Так как $u(x, T)$ - гладкая функция от $x \in \mathbb{R}^{n}$, то уравнение $\Delta v(x)=u_{0}(x)$ тоже должно иметь классические решения в шаре $|x|<r$. Но это противоречит выбору $u_{0}(x)$. Условие $\psi \in C^{2}\left(\mathbb{R}^{n}\right)$ не может вьполняться. Лемма доказана.

Для $u(x, t)$ из леммы 3 функция $\varphi(x)=T^{-1} \psi(x)$, вычисленная по формуле (2), также не принадлежит $C^{2}\left(\mathbb{R}^{n}\right)$. Теорема 1 оказывается неприменимой. Из предыдущих рассуждений ясно, что: 1$)$ поведение $u(x, t)$ на бесконечности не играет никакой роли; 2) возникший эффект связан исключительно с "несовершенством" оператора Лапласа $\Delta$ в пространстве $C\left(\mathbb{R}^{n}\right)$ - у уравнения Пуассона $\Delta v(x)=f(x)$ при специальном выборе непрерывной правой части $f(x)$ отсутствуют классические решения $[9 ;$ гл. IV,$\S 3]$. Фактически, это проявление незамкнутости $\Delta$ в пространстве $C\left(\mathbb{R}^{n}\right)$ на области определения $C^{2}\left(\mathbb{R}^{n}\right)$. Следующий параграф точнее разъяснит ситуацию.

\section{§5. Расширенный оператор Лапласа}

Построим замкнутое расширение оператора Лапласа $\Delta$ в пространстве $C\left(\mathbb{R}^{n}\right)$. Воспользуемся конструкцией И.И. Привалова [10] и введем расширенный оператор Лапласа $\Delta^{*}$ (обобщенный лапласиан, оператор Лапласа-Привалова) следующим образом.

При $n=1$ в $\mathbb{R}^{1}=\mathbb{R}$ положим $\Delta^{*} \equiv \Delta=d^{2} / d x^{2}$, понимая производную в классическом смысле. (Здесь достаточно стандартного определения.)

Пусть $n \geqslant 2$. Рассмотрим функцию $w \in C\left(\mathbb{R}^{n}\right)$. Для $r>0$ и $x \in \mathbb{R}^{n}$ обозначим

$$
\Delta_{r} w(x)=\frac{1}{b_{n} r^{n}} \int_{|y-x| \leqslant r} w(y) d y-w(x)=\frac{1}{b_{n} r^{n}} \int_{|y-x| \leqslant r}(w(y)-w(x)) d y,
$$

где $b_{n}=2 \pi^{n / 2}[n \Gamma(n / 2)]^{-1}$ - объем единичного шара в $\mathbb{R}^{n}$. Положим

$$
\Delta^{*} w(x)=\lim _{r \rightarrow 0}\left(\frac{2(n+2)}{r^{2}} \Delta_{r} w(x)\right)=\lim _{r \rightarrow 0} \frac{2(n+2)}{b_{n} r^{n+2}} \int_{|y-x| \leqslant r}(w(y)-w(x)) d y
$$

если этот предел существует. Вид предела (17) объясняется некоторыми простыми подсчетами, связанными с формулой Тейлора [10; ч. I, гл. I, § 1]. Перечислим основные свойства оператора $\Delta^{*}$.

1. Если $w \in C^{2}(\Omega)$ для области $\Omega \subset \mathbb{R}^{n}$, то $\Delta^{*} w(x)=\Delta w(x)$ всюду в $\Omega$. Таким образом, оператор $\Delta^{*}$ на функциях класса $C^{2}$ определен и совпадает с обычным оператором Лапласа [10; ч. I, гл. I, §1]. 
2. Если $\Delta^{*} w(x)=0$ в области $\Omega \subset \mathbb{R}^{n}$, то $w \in C^{2}(\Omega)$ и $\Delta w(x)=0$ всюду в $\Omega$. То есть непрерывное решение уравнения $\Delta^{*} w=0$ является обычной гармонической функцией [10; ч. I, гл. I, $\S 2]$.

3. Пусть $E(x)$ - фундаментальное решение уравнения Лапласа в $\mathbb{R}^{n}$ :

$$
E(x)=(2 \pi)^{-1} \ln |x|, \quad n=2 ; \quad E(x)=-\left((n-2) \omega_{n-1}\right)^{-1}|x|^{-(n-2)}, \quad n \geqslant 3 ;
$$

$\omega_{n-1}=2 \pi^{n / 2}[\Gamma(n / 2)]^{-1}-$ площадь поверхности единичной $(n-1)$-мерной сферы в $\mathbb{R}^{n}$. Для ограниченной области $\Omega \subset \mathbb{R}^{n}$ и функции $f \in C(\bar{\Omega})$ рассмотрим объемньй потенциал

$$
v(x)=\int_{\Omega} E(x-y) f(y) d y, \quad x \in \mathbb{R}^{n} .
$$

Тогда $\Delta^{*} v(x)=f(x)$ всюду в $\Omega$ [10; ч. II, гл. I, $\left.\S 1\right]$.

Данное свойство отличает оператор $\Delta^{*}$ от обычного оператора Лапласа. Хорошо известно, что потенциал (18) с произвольной непрерьвной плотностью $f$ может не удовлетворять (в классическом смысле) уравнению Пуассона $\Delta v(x)=f(x)$ во внутренних точках области $\Omega$ [11; гл. II, $\S 14]$.

Свойство 3 позволяет доказать следующие свойства 4,5 .

4. Пусть $\Delta^{*} w(x)=f(x)$ в области $\Omega \subset \mathbb{R}^{n}$, причем $f \in C^{k}(\Omega)$ с некоторым $k \in\{0,1,2, \ldots\}$. Тогда $w \in C^{k+1}(\Omega)$. Например, если $f \in C(\Omega)$, то $w \in C^{1}(\Omega)$; если $f \in C^{1}(\Omega)$, то $w \in C^{2}(\Omega)$ и $\Delta^{*} w(x)=\Delta w(x)$ в $\Omega$, и т.д.

Докажем свойство 4. Достаточно установить принадлежность $w \in C^{k+1}$ в произвольной гладкой ограниченной области, компактно вложенной в $\Omega$. Рассмотрим такую область и снова обозначим ее $\Omega$. Но теперь можно считать, что $f \in C^{k}(\bar{\Omega})$. Определим объемный потенциал $v(x)$ формулой (18). По свойству 3 получаем, что $\Delta^{*}(w(x)-v(x))=f(x)-f(x)=0$ в $\Omega$. Следовательно, $(w(x)-v(x))$ является гармонической функцией в $\Omega$ и гладкость $w(x)$ совпадает с гладкостью $v(x)$. Но гладкость потенциала $v(x)$ внутри $\Omega$ всегда хоть на единицу выше, чем гладкость плотности $f(x)$ (для $f \in C(\bar{\Omega})$ или $f \in C^{1}(\bar{\Omega})$ это классический факт; дальнейший переход легко осушествить по индукции при помощи формулы дифференцирования объемного потенциала, см. [9; гл. IV,$\S 3$, доказательство леммы 2]). Таким образом, $w \in C^{k+1}(\Omega)$. Свойство 4 доказано.

Как обычно, в гёльдеровых классах происходит повышение гладкости сразу на две единицы: если $f \in C^{k+\alpha}(\Omega)$ для некоторых $k \in\{0,1,2, \ldots\}$ и $\alpha \in(0,1)$, то $w \in C^{k+2+\alpha}(\Omega)$. Но такое уточнение нам не понадобится.

В качестве прямого следствия свойства 4 отметим, что всякая непрерывная собственная функция оператора $\Delta^{*}$ является обычной собственной функцией оператора Лапласа. Действительно, пусть $\Delta^{*} w(x)=\lambda w(x)$ в области $\Omega \subset \mathbb{R}^{n}$ при некотором $\lambda \in \mathbb{C}$ с непрерывной функцией $w(x)$. Последовательно применяя свойство 4 , получаем, что $w \in C^{1}(\Omega)$, затем, что $w \in C^{2}(\Omega)$, и тогда по свойству 1 $\Delta w(x)=\Delta^{*} w(x)=\lambda w(x)$, что и утверждалось.

Заключительное свойство 5 играет для нас ключевую роль. Оно и означает замкнутость оператора $\Delta^{*}$. 
5. Пусть $\Delta^{*} w_{\varepsilon}(x)=f_{\varepsilon}(x)$ в $\mathbb{R}^{n}$ для функций $w_{\varepsilon}, f_{\varepsilon} \in C\left(\mathbb{R}^{n}\right)$, отвечающих малому параметру $\varepsilon>0$. Если $w_{\varepsilon}(x) \rightarrow w_{0}(x)$ и $f_{\varepsilon}(x) \rightarrow f_{0}(x)$ при $\varepsilon \rightarrow 0$ равномерно на любом компакте в $\mathbb{R}^{n}$, то $\Delta^{*} w_{0}(x)$ сушествует всюду в $\mathbb{R}^{n}$ и $\Delta^{*} w_{0}(x)=f_{0}(x)$.

Докажем свойство 5 . Пусть $B$ - произвольный открытый шар в $\mathbb{R}^{n}$. Положим

$$
\left.v_{\varepsilon}(x)=\int_{B} E(x-y) f_{\varepsilon}(y) d y, \quad x \in \mathbb{R}^{n}, \quad \varepsilon \geqslant 0 \text { (включая } \varepsilon=0\right) .
$$

В силу свойства 3 для $\varepsilon>0$ все функции $w_{\varepsilon}(x)-v_{\varepsilon}(x)$ удовлетворяют в шаре $B$ уравнению $\Delta^{*}\left(w_{\varepsilon}(x)-v_{\varepsilon}(x)\right)=0$ и, значит, по свойству 2 являются гармоническими в $B$. При $\varepsilon \rightarrow 0$ имеем $\left(w_{\varepsilon}(x)-v_{\varepsilon}(x)\right) \rightarrow\left(w_{0}(x)-v_{0}(x)\right)$ равномерно в $\bar{B}$. Поэтому функция $w_{0}(x)-v_{0}(x)$ также является гармонической в $B$. Тем самым, $\Delta^{*}\left(w_{0}(x)-v_{0}(x)\right)=\Delta\left(w_{0}(x)-v_{0}(x)\right)=0$ всюду в $B$. Так как $v_{0}(x)$ есть объемный потенциал с непрерьвной плотностью $f_{0}(x)$, то $\Delta^{*} v_{0}(x)=f_{0}(x)$ в шаре $B$. Но тогда сушествует $\Delta^{*} w_{0}(x)=\Delta^{*} v_{0}(x)=f_{0}(x)$ в шаре $B$. Выбор $B$ был произвольньм. Свойство 5 доказано.

При $n=1$ в силу нашего определения $\Delta^{*}=\Delta=d^{2} / d x^{2}$ свойства 1,2 очевидны. Свойство 3 с фундаментальным решением $E(x)=|x| / 2$ проверяется непосредственно. Свойство 4 даже усиливается, а свойство 5 выводится из 3 так же, как при $n \geqslant 2$. Итак, свойства $1-5$ присущи оператору $\Delta^{*}$ при любом $n \geqslant 1$. Именно на эти свойства и только на них опираются все последующие рассуждения, связанные c $\Delta^{*}$.

Областью определения оператора $\Delta^{*}$ в пространстве $C\left(\mathbb{R}^{n}\right)$ считаем множество

$\mathscr{O}\left(\mathbb{R}^{n}\right) \equiv\left\{w \in C\left(\mathbb{R}^{n}\right): \Delta^{*} w(x)\right.$ сушествует всюду в $\mathbb{R}^{n}$ и $\left.\Delta^{*} w \in C\left(\mathbb{R}^{n}\right)\right\}$.

Из свойства 5 ясно, что $\Delta^{*}$ с указанной областью определения является замкнутьм оператором в $C\left(\mathbb{R}^{n}\right)$. С учетом свойства 1 можно утверждать, что $\Delta^{*}$ есть замкнутое расширение для $\Delta$. Отметим включения $C^{2}\left(\mathbb{R}^{n}\right) \subset \mathscr{O}\left(\mathbb{R}^{n}\right) \subset C^{1}\left(\mathbb{R}^{n}\right)$, следующие из свойств 1,4 . При $n=1$ справедливо равенство $\mathscr{O}\left(\mathbb{R}^{1}\right)=C^{2}\left(\mathbb{R}^{1}\right)$.

Дадим еще одно альтернативное описание для $\mathscr{O}\left(\mathbb{R}^{n}\right)$, имеющее "локальный" характер. Функция $w(x)$, определенная на $\mathbb{R}^{n}$, принадлежит $\mathscr{O}\left(\mathbb{R}^{n}\right)$ тог да и только тогда, когда в любой ограниченной области $\Omega \subset \mathbb{R}^{n}$ данная функция разлагается в сумму гармонической функции и некоторого объемного потенциала вида (18) с плотностью $f \in C(\bar{\Omega})$. При этом $f(x)=\Delta^{*} w(x)$ в $\Omega$.

Ввиду определения (19) и свойств $\Delta^{*}$ такая характеристика $\mathscr{O}\left(\mathbb{R}^{n}\right)$ очевидна.

\section{§ 6. Формулировка основного результата}

Введенное в (19) пространство $\mathscr{O}\left(\mathbb{R}^{n}\right)$ позволяет получить точное описание возможных правых частей $\varphi(x)$ в условии (2). Начнем со следующего утверждения.

ЛЕмма 4. Пусть $и(x, t)$ - любое решение уравнения теплопроводности (1) из класса $C^{2,1}\left(\mathbb{R}^{n} \times(0, T]\right) \cap C\left(\mathbb{R}^{n} \times[0, T]\right)$. При фиксированном $\tau \in(0, T]$ рассмотрим функцию

$$
\psi_{\tau}(x) \equiv \int_{0}^{\tau} u(x, t) d t, \quad x \in \mathbb{R}^{n} .
$$

Тогда $\psi_{\tau} \in \mathscr{O}\left(\mathbb{R}^{n}\right)$ и $\Delta^{*} \psi_{\tau}(x)=u(x, \tau)-u(x, 0)$ в $\mathbb{R}^{n}$. 
ДокАЗАТЕльство. Для $\varepsilon \in(0, \tau)$ запишем равенства $(15)$, заменив в крайней левой части $\Delta$ на $\Delta^{*}$ по свойству 1 оператора $\Delta^{*}$. Получим

$$
\Delta^{*} \int_{\varepsilon}^{\tau} u(x, t) d t=u(x, \tau)-u(x, \varepsilon), \quad x \in \mathbb{R}^{n}
$$

Устремим $\varepsilon$ к нулю, учитывая, что

$$
\int_{\varepsilon}^{\tau} u(x, t) d t \rightarrow \psi_{\tau}(x), \quad u(x, \tau)-u(x, \varepsilon) \rightarrow u(x, \tau)-u(x, 0)
$$

равномерно на любом компакте в $\mathbb{R}^{n}$. На основании свойства 5 оператора $\Delta^{*}$ заключаем, что $\psi_{\tau} \in \mathscr{O}\left(\mathbb{R}^{n}\right)$ и $\Delta^{*} \psi_{\tau}(x)=u(x, \tau)-u(x, 0)$ в $\mathbb{R}^{n}$. Лемма доказана.

Рассмотрим теперь задачу $(1),(2)$. Если $u(x, t)$ - ее решение, то функция $\varphi(x)$ в условии (2) совпадает с $T^{-1} \psi_{T}(x)$, где функция $\psi_{T}(x) \equiv \psi(x)$ построена при $\tau=T$ по формуле (20) (или просто по формуле (3)). Из леммы 4 следует, что $\varphi \in \mathscr{O}\left(\mathbb{R}^{n}\right)$ и $\Delta^{*} \varphi(x)=T^{-1}(u(x, T)-u(x, 0))$ в $\mathbb{R}^{n}$.

Итак, для получения разрешимой задачи $(1),(2)$ функцию $\varphi(x)$ в условии $(2)$ необходимо выбирать в $\mathscr{O}\left(\mathbb{R}^{n}\right)$, т.е. для нее должен быть корректно определен расширенньй оператор Лапласа $\Delta^{*} \varphi(x)$ такой, что $\Delta^{*} \varphi \in C\left(\mathbb{R}^{n}\right)$. Как известно из леммы 3 , обычный оператор $\Delta$ для $\varphi(x)=T^{-1} \psi(x)$ может быть не определен.

Формула (11) для начального состояния $u_{0}(x)$ приобретает вид

$$
u_{0}(x)=\int_{\mathbb{R}^{n}} g_{T}(x-y) \varphi(y) d y-T \Delta^{*} \varphi(x), \quad x \in \mathbb{R}^{n}
$$

Сформулируем уточненный результат о разрешимости задачи (1), (2). Он включает в себя предыдущую теорему 1.

Теорема 2. Для того чтобъ задача (1), (2) с функиией $\varphi(x)$ бъла разрешимой в классе $\mathscr{E}_{\sigma}\left(\mathbb{R}^{n} \times[0, T]\right)$ при некотором фиксированном $\sigma,|\sigma|<\sqrt{\pi / T}$, необходимо и достаточно, чтобы $\varphi \in \mathscr{O}\left(\mathbb{R}^{n}\right) \cap \mathscr{E}_{\sigma}\left(\mathbb{R}^{n}\right)$ и $\Delta^{*} \varphi \in \mathscr{E}_{\sigma}\left(\mathbb{R}^{n}\right)$. Для любой такой $\varphi(x)$ решение $и(x, t)$ задачи $(1),(2)$, принадлежащее $\mathscr{E}_{\sigma}\left(\mathbb{R}^{n} \times[0, T]\right)$, будет единственным. Оно находится по формулам (4), (21), причем функция $g_{T}(x)$ в (21) определяется формулой (10).

Теоремы 1, 2 легко обозримы и потому удобны для приложений. Мы получим их как следствия более полного основного утверждения, учитывающего практически все возможности. Для краткости используем обозначение $\sigma_{0} \equiv \sqrt{\pi / T}$.

Теорема 3. Пусть $\sigma$ - фиксированное число, $-\infty<\sigma<\sigma_{0} \equiv \sqrt{\pi / T}$. Справедливь следующие утвержсдения:

1) при любой функиии $\varphi \in C\left(\mathbb{R}^{n}\right)$ задача (1), (2) в классе $\mathscr{E}_{\sigma}\left(\mathbb{R}^{n} \times[0, T]\right)$ может иметь не более одного решения;

2) для того чтобы задача (1), (2) бълла разрешимой в $\mathscr{E}_{\sigma}\left(\mathbb{R}^{n} \times[0, T]\right)$, необходимо, чтобы $\varphi \in \mathscr{O}\left(\mathbb{R}^{n}\right) \cap \mathscr{E}_{\sigma}\left(\mathbb{R}^{n}\right)$ и $\Delta^{*} \varphi \in \mathscr{E}_{\sigma}\left(\mathbb{R}^{n}\right) ;$ 
3) обратно, пусть $\varphi \in \mathscr{O}\left(\mathbb{R}^{n}\right) \cap \mathscr{E}_{\sigma}\left(\mathbb{R}^{n}\right) u \Delta^{*} \varphi \in \mathscr{E}_{\sigma}\left(\mathbb{R}^{n}\right)$, m.e.

$$
|\varphi(x)| \leqslant M \exp (\sigma|x|), \quad\left|\Delta^{*} \varphi(x)\right| \leqslant M \exp (\sigma|x|), \quad x \in \mathbb{R}^{n},
$$

c некоторой константой $M>0$. Тогда задача (1), (2) имеет решение $u(x, t)$, которое находится по формулам (4), (21), причем функиия $g_{T}(x)$ в (21) определяется формулой (10). Дальнейшее описание данного решения $u(x, t)$ зависит от того, в какой промежуток попадает исходное значение $\sigma$;

4) при $-\sigma_{0}<\sigma<\sigma_{0}$ решение $u(x, t)$ принадлежит $\mathscr{E}_{\sigma}\left(\mathbb{R}^{n} \times[0, T]\right) u$ удовлетворяет оценке устойчивости

$$
|u(x, t)| \leqslant M N \exp (\sigma|x|), \quad x \in \mathbb{R}^{n}, \quad 0 \leqslant t \leqslant T,
$$

где $M$ такая жсе, как в (22), а константа $N>0$ зависит только от $n, T, \sigma$, но не зависит от $M u \varphi(x)$;

5) при $\sigma<-\sigma_{0}$ решение $u(x, t)$ принадлежит $\mathscr{E}_{-\sigma_{0}}\left(\mathbb{R}^{n} \times[0, T]\right)$ и удовлетворяет оценке устойчивости

$$
|u(x, t)| \leqslant M N \exp \left(-\sigma_{0}|x|\right), \quad x \in \mathbb{R}^{n}, \quad 0 \leqslant t \leqslant T,
$$

где $M$ такая жсе, как в (22), а константа $N>0$ зависит только от $n, T, \sigma$

6) наконеи, при $\sigma=-\sigma_{0}$ можнно утверждать, что

$$
u \in \bigcap_{\sigma_{1}>-\sigma_{0}} \mathscr{E}_{\sigma_{1}}\left(\mathbb{R}^{n} \times[0, T]\right),
$$

причем для любого $\sigma_{1}>-\sigma_{0}$ справедлива оченка

$$
|u(x, t)| \leqslant M N \exp \left(\sigma_{1}|x|\right), \quad x \in \mathbb{R}^{n}, \quad 0 \leqslant t \leqslant T,
$$

где $M$ такая жее, как в (22), а константа $N>0$ зависит только от $n, T, \sigma_{1}$.

Теоремы 1, 2 непосредственно следуют из общей теоремы 3, поэтому будем доказывать только ее. Прежде чем приступить к доказательству ${ }^{3}$, изучим асимптотическое поведение функции $g_{T}(x)$, входящей в $(21)$.

\section{§7. Исследование $g_{T}(x)$}

Получим вначале разложение $g_{T}(x)$ в сходящийся ряд по функциям Ханкеля, а затем выведем нужные оценки на бесконечности. Напомним, что всюду в статье символ $n$ означает размерность основного пространства $\mathbb{R}^{n} ; x=\left(x_{1}, \ldots, x_{n}\right) \in \mathbb{R}^{n}$. Кроме того, в данном параграфе используется обозначение $\nu \equiv(n-2) / 2$. Сведения по специальным функциям, приводимые без ссылок, см. в [12], [13].

\footnotetext{
3 Доказательство основной теоремы 3 см. в $\S 8$; дополнительный комментарий к пा. 4)-6) дан в $\S 9$; некоторые специальные уточнения сделаны в $§ 10$.
} 
Теорема 4. Для функиии $g_{T}(x)$, определенной на $\mathbb{R}^{n}$ формулой (10), справедливо разложение в ряд

$$
g_{T}(x)=-2(\pi|x|)^{-\nu} \sum_{k=1}^{\infty} \operatorname{Im}\left(\left(\frac{z_{k}}{2}\right)^{\nu+2} H_{\nu}^{(1)}\left(z_{k}|x|\right)\right),
$$

сходящийся при $|x| \neq 0$. Здесь $\nu \equiv(n-2) / 2, z_{k} \equiv(1+i) \sqrt{k \pi / T}, H_{\nu}^{(1)}-$ первая функиия Ханкеля индекса $\nu$.

ЗАмечание 1. Прокомментируем формулу (27). Мы записали ее в виде, наиболее удобном для рассмотрений. Возможны "косметические" изменения, связанные с другими группировками сомножителей. В случае нечетного $n$, когда степень $\nu+2=(n+2) / 2$ является полуцелой, значение $\left(z_{k} / 2\right)^{\nu+2} H_{\nu}^{(1)}\left(z_{k}|x|\right)$ для комплексных $z_{k}=(1+i) \sqrt{k \pi / T}$ вычисляется так. Учитывая, что индекс $\nu=(n-2) / 2$ тоже полуцелый, выразим $H_{\nu}^{(1)}$ через функции Бесселя $J_{\nu}, J_{-\nu}$ по формуле

$$
H_{\nu}^{(1)}(z) \equiv(i \sin \nu \pi)^{-1}\left[J_{-\nu}(z)-\exp (-\nu \pi i) J_{\nu}(z)\right]=J_{\nu}(z)-i^{2 \nu} J_{-\nu}(z) .
$$

Умножим на $(z / 2)^{\nu+d}$ с натуральным $d$ и перейдем к степенньп рядам:

$$
\left(\frac{z}{2}\right)^{\nu+d} H_{\nu}^{(1)}(z)=\sum_{m=0}^{\infty} \frac{(-1)^{m}(z / 2)^{2 m+2 \nu+d}}{m ! \Gamma(m+\nu+1)}-i^{2 \nu} \sum_{m=0}^{\infty} \frac{(-1)^{m}(z / 2)^{2 m+d}}{m ! \Gamma(m-\nu+1)} .
$$

Здесь $2 \nu=n-2$ - целое число, $2 \nu+d=n+d-2$ - неотрицательное целое число. Поэтому (28) определяет целую функцию $(z / 2)^{\nu+d} H_{\nu}^{(1)}(z)$. Можно показать, что это элементарная функция, точнее, квазиполином вида $z^{d-1} p_{\nu}(z) \exp (i z)$, где полином $p_{\nu}(z)$ зависит только от $\nu$ (см. [12; формулы 7.11.(3) и 7.11.(17)]). В частности, при $d=2$ и $z=z_{k}|x|$ значение

$$
\left(\frac{z_{k}}{2}\right)^{\nu+2} H_{\nu}^{(1)}\left(z_{k}|x|\right)=|x|^{-(\nu+2)}\left(\frac{z_{k}|x|}{2}\right)^{\nu+2} H_{\nu}^{(1)}\left(z_{k}|x|\right)
$$

однозначно вычисляется согласно (28). Вообще условимся, что потенциально многозначные функции типа $z^{\alpha}, J_{\alpha}(z), H_{\alpha}^{(1)}(z)$ и т.д. всегда рассматриваются только в плоскости $\mathbb{C}$ с разрезом $(-\infty, 0]$ для главного значения аргумента $z,-\pi<$ $\arg z<\pi$. Тогда эти функции определяются своими стандартными формулами однозначно.

ДОКАЗАТЕЛЬСТВО ТЕОРЕМЫ 4. В последующих выкладках используется функция

$$
F_{T}(s) \equiv \frac{T s^{2}}{\exp \left(T s^{2}\right)-1}
$$

для вешественных или комплексных $s$. Заметим, что $G_{T}(\xi)=F_{T}(|\xi|)$ для функции $G_{T}(\xi)$ из формулы $(9)$. Обозначим $|\xi|=r$ и преобразуем $(10)$, интегрируя сначала по сферам радиуса $r$, а затем по $r \in(0, \infty)$. Получим

$$
\begin{aligned}
g_{T}(x) & =\frac{1}{(2 \pi)^{n}} \int_{0}^{\infty} F_{T}(r) d r \int_{|\xi|=r} \exp (i \xi x) d S_{\xi} \\
& =\frac{1}{(2 \pi)^{n}} \int_{0}^{\infty} F_{T}(r) r^{n-1} d r \int_{|\xi|=1} \exp (i r \xi x) d S_{\xi} .
\end{aligned}
$$


Для точности формулировок считаем $|x| \neq 0$ (хотя многие последующие соотношения справедливы при $|x|=0$ в “предельном" смысле). Согласно [14; п. 4.3.2.5] при интегрировании по единичной сфере в $\mathbb{R}^{n}$ имеет место формула

$$
\int_{|\xi|=1} \exp (i r \xi x) d S_{\xi}=(2 \pi)^{n / 2}(r|x|)^{-(n-2) / 2} J_{(n-2) / 2}(r|x|)
$$

Формула верна и при $n=1$ : надо понимать интеграл как $\exp (\operatorname{ir} x)+\exp (-\operatorname{ir} x)$ и заметить, что $J_{-1 / 2}(z)=\sqrt{2 /(\pi z)} \cos z$. Таким образом, при любом $n$ вьполняется равенство

$$
g_{T}(x)=(2 \pi)^{-n / 2}|x|^{-(n-2) / 2} \int_{0}^{\infty} F_{T}(r) r^{n / 2} J_{(n-2) / 2}(r|x|) d r
$$

Переменная интегрирования $r$ утратила свой “физический” смысл, удобно обозначить ее через $s$. Полагая $\nu=(n-2) / 2$ и учитывая, что $J_{\nu}(s|x|)=\operatorname{Re} H_{\nu}^{(1)}(s|x|)$ при $s>0$, перепишем (29) в виде

$$
g_{T}(x)=\pi^{-(\nu+1)}|x|^{-\nu} \operatorname{Re} \int_{0}^{\infty}\left(\frac{s}{2}\right)^{\nu+1} H_{\nu}^{(1)}(s|x|) F_{T}(s) d s .
$$

(Как ясно из следующего абзаца, подынтегральное выражение в (30) не имеет особенности в нуле, интеграл сходится и потому знак Re можно вынести из-под интеграла ${ }^{4}$.) Разложение (27) получим, интегрируя функцию $(s / 2)^{\nu+1} H_{\nu}^{(1)}(s|x|) F_{T}(s)$ не по лучу $[0, \infty)$, а по специальным контурам в комплексной плоскости.

Контуры принадлежат первому координатному углу

$$
\mathbb{C}_{++} \equiv\{z: \operatorname{Re} z \geqslant 0, \operatorname{Im} z \geqslant 0\}
$$

Функция $(z / 2)^{\nu+1} H_{\nu}^{(1)}(z)$ голоморфна внутри $\mathbb{C}_{++}$и непрерьвна вплоть до граниџы. При полуцелом $\nu$ (когда $n$ нечетно) это следует из представления $(28)$, взятого с $d=1$. Здесь функция $(z / 2)^{\nu+1} H_{\nu}^{(1)}(z)$ является целой. При целом $\nu$ (когда $n$ четно) надо учесть, что функции Ханкеля голоморфны в $\mathbb{C}$ с разрезом $(-\infty, 0]$, причем при $z \rightarrow 0$ справедливы оценки $H_{\nu}^{(1)}(z)=O\left(|z|^{-\nu}\right), \nu \neq 0$, и $H_{0}^{(1)}(z)=O(\ln |z|)$, которые гарантируют непрерывность в нуле функции $(z / 2)^{\nu+1} H_{\nu}^{(1)}(z)$ (см. [13; формулы 9.1.8 и 9.1.9]). Аналогично, функция

$$
\left(\frac{z}{2}\right)^{\nu+1} H_{\nu}^{(1)}(z|x|)=|x|^{-(\nu+1)}\left(\frac{z|x|}{2}\right)^{\nu+1} H_{\nu}^{(1)}(z|x|)
$$

при фиксированном $|x| \neq 0$ голоморфна внутри $\mathbb{C}_{++}$и непрерывна вплоть до границы.

\footnotetext{
${ }^{4}$ Сходимость интеграла "на бесконечности" очевидно следует из быстрого стремления к нулю функции $F_{T}(s)$ при $s \rightarrow+\infty$.
} 
Функция $F_{T}(z)=T z^{2}\left(\exp \left(T z^{2}\right)-1\right)^{-1}$ мероморфина в $\mathbb{C}$. В угол $\mathbb{C}_{++}$попадают ее простые полюсы $z_{k}=(1+i) \sqrt{k \pi / T}, k \in \mathbb{N}$.

Фиксируем числа $a_{m} \equiv \sqrt{(m+0.5) \pi / T}, m \in \mathbb{N}$, и для каждого $m$ рассмотрим прямоугольник

$$
\Pi_{m} \equiv\left\{z: 0 \leqslant \operatorname{Re} z \leqslant 2 a_{m}, 0 \leqslant \operatorname{Im} z \leqslant a_{m}\right\}
$$

содержащий точки $z_{k} \mathrm{c} k=1, \ldots, m$. По теореме о вычетах

$$
\begin{aligned}
\int_{\partial \Pi_{m}}\left(\frac{z}{2}\right)^{\nu+1} H_{\nu}^{(1)}(z|x|) F_{T}(z) d z & =2 \pi i \sum_{k=1}^{m} \operatorname{Res}_{z=z_{k}} \frac{(z / 2)^{\nu+1} H_{\nu}^{(1)}(z|x|) T z^{2}}{\exp \left(T z^{2}\right)-1} \\
& =2 \pi i \sum_{k=1}^{m}\left(\frac{z_{k}}{2}\right)^{\nu+2} H_{\nu}^{(1)}\left(z_{k}|x|\right) .
\end{aligned}
$$

Построим контур $L_{m}$, состоящий из трех отрезков $L_{m}^{(1)}=\left\{i \tau: 0 \leqslant \tau \leqslant a_{m}\right\}$, $L_{m}^{(2)}=\left\{s+i a_{m}: 0 \leqslant s \leqslant 2 a_{m}\right\}, L_{m}^{(3)}=\left\{2 a_{m}+i \tau: a_{m} \geqslant \tau \geqslant 0\right\}$ и луча $L_{m}^{(4)}=$ $\left[2 a_{m}, \infty\right)$ положительной полуоси. Определим последовательность интегралов

$$
\Upsilon_{T, m}(x) \equiv \int_{L_{m}}\left(\frac{z}{2}\right)^{\nu+1} H_{\nu}^{(1)}(z|x|) F_{T}(z) d z, \quad m \in \mathbb{N}
$$

Так как $\partial \Pi_{m}$ является объединением отрезков $\left[0,2 a_{m}\right]$ и $L_{m}^{(j)}, j=1,2,3$, то

$$
\int_{0}^{\infty}\left(\frac{s}{2}\right)^{\nu+1} H_{\nu}^{(1)}(s|x|) F_{T}(s) d s=\Upsilon_{T, m}(x)+\int_{\partial \Pi_{m}}\left(\frac{z}{2}\right)^{\nu+1} H_{\nu}^{(1)}(z|x|) F_{T}(z) d z .
$$

Учитывая (30), а затем (31), приходим к равенствам

$$
\begin{aligned}
\pi^{\nu+1}|x|^{\nu} g_{T}(x) & =\operatorname{Re} \Upsilon_{T, m}(x)+\operatorname{Re} \int_{\partial \Pi_{m}}\left(\frac{z}{2}\right)^{\nu+1} H_{\nu}^{(1)}(z|x|) F_{T}(z) d z \\
& =\operatorname{Re} \Upsilon_{T, m}(x)-2 \pi \operatorname{Im} \sum_{k=1}^{m}\left(\frac{z_{k}}{2}\right)^{\nu+2} H_{\nu}^{(1)}\left(z_{k}|x|\right) .
\end{aligned}
$$

Теперь при фиксированном $x,|x| \neq 0$, перейдем к пределу в $(32)$ по $m \rightarrow \infty$. Сами интегралы $\Upsilon_{T, m}(x)$ могут не стремиться к нулю, но мы докажем, что этим свойством обладают их действительные части.

Напомним (см. [15; столбец 870]), что функция $(z / 2)^{\nu+1} H_{\nu}^{(1)}(z)$ принимает на верхней мнимой полуоси лишь вещественные значения ${ }^{5}$. Очевидно, что тем же

\footnotetext{
${ }^{5}$ Это следует, например, из формулы $(i s / 2)^{\nu+1} H_{\nu}^{(1)}(i s)=2 \pi^{-1}(s / 2)^{\nu+1} K_{\nu}(s), s>0$, связывающей функцию Ханкеля $H_{\nu}^{(1)}$ с функцией Макдональда $K_{\nu}$. Как известно, при $s>0$ значения $K_{\nu}(s)$ являются вещественньпи (см. [13; п. 9.6]).
} 
свойством обладают функции $(z / 2)^{\nu+1} H_{\nu}^{(1)}(z|x|)$ и $F_{T}(z)$. Поэтому интеграл, входящий в $\Upsilon_{T, m}(x)$, по отрезку $L_{m}^{(1)}$ мнимой оси - чисто мнимая величина. Но тогда $\operatorname{Re} \Upsilon_{T, m}(x)=\operatorname{Re} \sum_{j=2}^{4} \Upsilon_{T, m}^{(j)}(x)$, где

$$
\Upsilon_{T, m}^{(j)}(x) \equiv \int_{L_{m}^{(j)}}\left(\frac{z}{2}\right)^{\nu+1} H_{\nu}^{(1)}(z|x|) F_{T}(z) d z
$$

Оценим сверху модуль каждого из интегралов $\Upsilon_{T, m}^{(j)}(x), j=2,3,4$.

При $j=2$ имеем

$$
\begin{aligned}
\left|\Upsilon_{T, m}^{(2)}(x)\right| & \leqslant 2 a_{m} \max _{z \in L_{m}^{(2)}}\left(\left|\frac{z}{2}\right|^{\nu+1}\left|H_{\nu}^{(1)}(z|x|)\right| \frac{T|z|^{2}}{\left|\exp \left(T z^{2}\right)-1\right|}\right) \\
& \leqslant 2^{-\nu} a_{m}\left(a_{m} \sqrt{5}\right)^{\nu+3} T \max _{0 \leqslant s \leqslant 2 a_{m}}\left|H_{\nu}^{(1)}\left(\left(s+i a_{m}\right)|x|\right)\right| \cdot\left(C_{m}^{(2)}\right)^{-1}
\end{aligned}
$$

где

$$
C_{m}^{(2)} \equiv \min _{z \in L_{m}^{(2)}}\left|\exp \left(T z^{2}\right)-1\right| \geqslant \frac{1}{2}, \quad m \in \mathbb{N} .
$$

Элементарные неравенства (33) докажем чуть позже, а пока отметим, что согласно оценкам Вебера [16; п. 7.33] для функции $H_{\nu}^{(1)}(z)$ вьполняется соотношение

$$
\left|H_{\nu}^{(1)}(z)\right| \leqslant \kappa_{\nu}|z|^{-1 / 2} \exp (-\operatorname{Im} z), \quad z \in \mathbb{C}_{++}, \quad|z| \geqslant 1,
$$

с некоторой константой $\kappa_{\nu}>0$, зависящей только от $\nu$. В частности,

$$
\max _{0 \leqslant s \leqslant 2 a_{m}}\left|H_{\nu}^{(1)}\left(\left(s+i a_{m}\right)|x|\right)\right| \leqslant \kappa_{\nu}\left(a_{m}|x|\right)^{-1 / 2} \exp \left(-a_{m}|x|\right), \quad a_{m}|x| \geqslant 1
$$

Воспользовавшись (33) и (35), получим

$$
\left|\Upsilon_{T, m}^{(2)}(x)\right| \leqslant M_{\nu}^{(2)} T|x|^{-1 / 2} a_{m}^{\nu+3.5} \exp \left(-a_{m}|x|\right), \quad a_{m}|x| \geqslant 1
$$

с константой $M_{\nu}^{(2)}>0$, зависящей только от $\nu$.

Для окончательного обоснования (36) осталось доказать неравенства (33). Рассмотрим $z=s+i a_{m}, 0 \leqslant s \leqslant 2 a_{m}$. Заметим, что

$$
\exp \left(T z^{2}\right)=\exp \left(T\left(s^{2}-a_{m}^{2}\right)\right)\left(\cos \left(2 T a_{m} s\right)+i \sin \left(2 T a_{m} s\right)\right)
$$

Здесь $a_{m} \equiv \sqrt{(m+0.5) \pi / T}$. Если $s \in\left(a_{m}-\pi /\left(4 a_{m} T\right), a_{m}+\pi /\left(4 a_{m} T\right)\right)$, то $2 T a_{m} s \in(2 m \pi+\pi / 2,2 m \pi+3 \pi / 2)$. При этих значениях $s$ вешественная часть в $(37)$ отрицательна и потому $\left|\exp \left(T z^{2}\right)-1\right|>1$. Если же $\left|s-a_{m}\right| \geqslant \pi /\left(4 a_{m} T\right)$, TO

$$
T\left|s^{2}-a_{m}^{2}\right|=T\left|s-a_{m}\right|\left|s+a_{m}\right| \geqslant T\left|s-a_{m}\right| a_{m} \geqslant \frac{\pi}{4}>\frac{3}{4},
$$


откуда $\left|\exp \left(T z^{2}\right)\right|=\exp \left(T\left(s^{2}-a_{m}^{2}\right)\right) \in(0, \exp (-3 / 4)) \cup(\exp (3 / 4),+\infty)$. Но тогда

$$
\left|\exp \left(T z^{2}\right)-1\right| \geqslant|| \exp \left(T z^{2}\right)|-1| \geqslant 1-\exp \left(-\frac{3}{4}\right)>\frac{1}{2}
$$

Итак, неравенства (33) выполнены, соотношение (36) обосновано. Из (36) следует, что $\Upsilon_{T, m}^{(2)}(x) \rightarrow 0$ при $m \rightarrow \infty$ для любого фиксированного $x,|x|>0$.

Перейдем к $\Upsilon_{T, m}^{(3)}(x)$. Здесь $L_{m}^{(3)}$ - вертикальный отрезок $\left[2 a_{m}+i a_{m}, 2 a_{m}\right]$. Его длина равна $a_{m}$. Функция $|z|$ принимает на $L_{m}^{(3)}$ максимальное значение $a_{m} \sqrt{5}$. Сравнивая с предыдущим, получаем

$$
\left|\Upsilon_{T, m}^{(3)}(x)\right| \leqslant 2^{-(\nu+1)} a_{m}\left(a_{m} \sqrt{5}\right)^{\nu+3} T \max _{0 \leqslant \tau \leqslant a_{m}}\left|H_{\nu}^{(1)}\left(\left(2 a_{m}+i \tau\right)|x|\right)\right| \cdot\left(C_{m}^{(3)}\right)^{-1},
$$

$\Gamma$ де 6

$$
\begin{aligned}
C_{m}^{(3)} & \equiv \min _{z \in L_{m}^{(3)}}\left|\exp \left(T z^{2}\right)-1\right| \geqslant \min _{0 \leqslant \tau \leqslant a_{m}}\left|\exp \left(T\left(2 a_{m}+i \tau\right)^{2}\right)\right|-1 \\
& =\min _{0 \leqslant \tau \leqslant a_{m}} \exp \left(T\left(4 a_{m}^{2}-\tau^{2}\right)\right)-1=\exp \left(3 T a_{m}^{2}\right)-1 .
\end{aligned}
$$

Поскольку $3 T a_{m}^{2}=3(m+0.5) \pi \geqslant 9 \pi / 2>1>\ln 2$ при $m \in \mathbb{N}$, то

$$
\left(C_{m}^{(3)}\right)^{-1} \leqslant \frac{\exp \left(-3 T a_{m}^{2}\right)}{1-\exp \left(-3 T a_{m}^{2}\right)} \leqslant \frac{\exp \left(-3 T a_{m}^{2}\right)}{1-2^{-1}}=2 \exp \left(-3 T a_{m}^{2}\right)
$$

Согласно (34) справедлива оценка

$$
\max _{0 \leqslant \tau \leqslant a_{m}}\left|H_{\nu}^{(1)}\left(\left(2 a_{m}+i \tau\right)|x|\right)\right| \leqslant \kappa_{\nu}\left(2 a_{m}|x|\right)^{-1 / 2}, \quad 2 a_{m}|x| \geqslant 1 .
$$

Воспользовавшись (38) и (39), заключаем, что

$$
\left|\Upsilon_{T, m}^{(3)}(x)\right| \leqslant M_{\nu}^{(3)} T|x|^{-1 / 2} a_{m}^{\nu+3.5} \exp \left(-3 T a_{m}^{2}\right), \quad a_{m}|x| \geqslant \frac{1}{2}
$$

с константой $M_{\nu}^{(3)}>0$, зависяшей только от $\nu$. Из $(40)$ ясно, что $\Upsilon_{T, m}^{(3)}(x) \rightarrow 0$ при $m \rightarrow \infty$ для любого фиксированного $x,|x|>0$.

Рассмотрим, наконец, интеграл $\Upsilon_{T, m}^{(4)}(x)$ по лучу $L_{m}^{(4)}=\left[2 a_{m}, \infty\right)$ вещественной оси. Вновь используя (34), получаем оценку

$$
\left|\Upsilon_{T, m}^{(4)}(x)\right| \leqslant 2^{-(\nu+1)} \kappa_{\nu}|x|^{-1 / 2} T \int_{2 a_{m}}^{\infty} \frac{s^{\nu+2.5}}{\exp \left(T s^{2}\right)-1} d s, \quad 2 a_{m}|x| \geqslant 1 .
$$

\footnotetext{
${ }^{6}$ В следующей выкладке (и только в ней) используется то, что в качестве $\Pi_{m}$ удобно было взять прямоугольник со сторонами $2 a_{m} \times a_{m}$, а не квадрат со сторонами $a_{m} \times a_{m}$.
} 
Функция $s^{\nu+2.5}\left(\exp \left(T s^{2}\right)-1\right)^{-1}$ суммируема на $[0, \infty)$. Последовательность интегралов по лучам $\left[2 a_{m}, \infty\right)$ стремится к нулю при $m \rightarrow \infty$. Таким образом, $\Upsilon_{T, m}^{(4)}(x) \rightarrow 0$ при $m \rightarrow \infty$ для любого фиксированного $x,|x|>0$.

$\mathrm{B}$ итоге, $\operatorname{Re} \Upsilon_{T, m}(x)=\operatorname{Re} \sum_{j=2}^{4} \Upsilon_{T, m}^{(j)}(x) \rightarrow 0$ при $m \rightarrow \infty$ для любого фиксированного $x,|x|>0$. Переходя к пределу $m \rightarrow \infty$ в (32), получаем разложение (27). Сходимость ряда $(27)$ к $g_{T}(x)$ при $|x|>0$ полностью обоснована. Теорема 4 доказана.

Теорема 4 имеет, на наш взгляд, принципиальный характер. Используя разложение (27) и асимптотику функций Ханкеля в комплексной области, можно получать различные оценки для $g_{T}(x)$ при $|x| \rightarrow \infty$. Соответствующие результаты оформлены далее в виде лемм (см. леммы 6, 7 ниже). Предварительно удобно доказать элементарную лемму 5 , связанную с одним асимптотическим разложением. Теория асимптотических разложений, как таковая, затрагивается здесь в минимальной степени; необходимые определения см. в [15; столбцы 337-338], [17; гл. I, $\S 2]$.

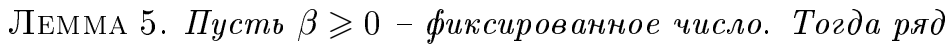

$$
\sum_{k=1}^{\infty} k^{\beta} \exp (-\rho \sqrt{k})
$$

сходится при всех $\rho>0$ и при $\rho \rightarrow+\infty$ является асимптотическим разложением для своей суммы $S(\rho)$ по последовательности $\{\exp (-\rho \sqrt{k})\}_{k=1}^{\infty}$. Всякий остаток ряда оценивается через свое первое слагаемое по формуле

$$
\sum_{k=m}^{\infty} k^{\beta} \exp (-\rho \sqrt{k}) \leqslant 2 m^{\beta} \exp (-\rho \sqrt{m}), \quad \rho \geqslant \rho_{\beta, m}
$$

с константой $\rho_{\beta, m}>0$, зависящей только от $\beta$ и т. В частности, для суммы ряда $S(\rho)$ справедлива оченка

$$
S(\rho) \equiv \sum_{k=1}^{\infty} k^{\beta} \exp (-\rho \sqrt{k}) \leqslant 2 \exp (-\rho), \quad \rho \geqslant \rho_{\beta},
$$

где $\rho_{\beta}>0$ зависит только от $\beta$.

ДокАЗАТЕЛЬСТво. Функция $h_{\rho}(s) \equiv s^{\beta} \exp (-\rho \sqrt{s})$ с параметром $\rho>0$ положительна при $s \geqslant 0$ и монотонно стремится к нулю при $s>(2 \beta / \rho)^{2}$. Интеграл от $h_{\rho}(s)$ по промежутку $0 \leqslant s<\infty$ конечен - он равен $2 \rho^{-(2 \beta+2)} \Gamma(2 \beta+2)$. Применяя интегральньй признак Коши, заключаем, что ряд $\sum_{k=1}^{\infty} h_{\rho}(k)$, т.е. ряд $(41)$, сходится при любом $\rho>0$ к некоторой конечной сумме $S(\rho)$. Рассмотрим $m$-й остаток

$$
R_{m}(\rho) \equiv S(\rho)-\sum_{k=1}^{m} k^{\beta} \exp (-\rho \sqrt{k})=\exp (-\rho \sqrt{m}) \sum_{k=m+1}^{\infty} k^{\beta} \exp (-\rho(\sqrt{k}-\sqrt{m}))
$$


Пусть $\rho>2 \beta m^{-1 / 2}$. Тогда функция $s^{\beta} \exp (-\rho(\sqrt{s}-\sqrt{m}))=\exp (\rho \sqrt{m}) h_{\rho}(s)$ переменной $s$ заведомо убьвает при $s \geqslant m$ и

$$
\sum_{k=m+1}^{\infty} k^{\beta} e^{-\rho(\sqrt{k}-\sqrt{m})} \leqslant \int_{m}^{\infty} s^{\beta} e^{-\rho(\sqrt{s}-\sqrt{m})} d s=2 \int_{0}^{\infty}(\tau+\sqrt{m})^{2 \beta+1} e^{-\rho \tau} d \tau .
$$

Последний интеграл очевидно стремится к нулю при $\rho \rightarrow+\infty$. Тем самым,

$$
R_{m}(\rho)=o(\exp (-\rho \sqrt{m})), \quad \rho \rightarrow+\infty .
$$

По определению [17; гл. I, §2] ряд (41) при $\rho \rightarrow+\infty$ является асимптотическим разложением для $S(\rho)$ (в смысле Пуанкаре) по асимптотической последовательности $\{\exp (-\rho \sqrt{k})\}_{k=1}^{\infty}$. Для получения (42) достаточно заметить, что фигурируюший там остаток ряда имеет вид $m^{\beta} \exp (-\rho \sqrt{m})+R_{m}(\rho)$, где $R_{m}(\rho)=$ $o(\exp (-\rho \sqrt{m}))$ при $\rho \rightarrow+\infty$. Отсюда (42) сразу и следует. Оценка (43) есть частньй случай (42) при $m=1$. Лемма доказана.

Итак, сумму $S(\rho)$ ряда (41) можно оценить при помощи первого слагаемого по формуле (43). Применим этот принцип к функции $g_{T}(x)$.

Лемма 6. Для функции $g_{T}(x)$, определенной формулой (10), справедлива оценка

$$
\left|g_{T}(x)\right| \leqslant C_{T, n}|x|^{-(n-1) / 2} \exp \left(-\sqrt{\frac{\pi}{T}}|x|\right), \quad x \in \mathbb{R}^{n} \backslash\{0\},
$$

с константой $C_{T, n}>0$, зависящей лишь от $T$ и размерности $n$ пространства $\mathbb{R}^{n}$. В частности,

$$
\left|g_{T}(x)\right| \leqslant D_{T, n} \exp \left(-\sqrt{\frac{\pi}{T}}|x|\right), \quad x \in \mathbb{R}^{n},
$$

с константой $D_{T, n}>0$, зависящей лишь от $T, n$. При әтом можно положить $C_{T, n}=T^{-(n+1) / 4} C_{1, n}$ и $D_{T, n}=T^{-n / 2} D_{1, n}$, где $C_{1, n}$ и $D_{1, n}$ - значения констант при $T=1$, зависящие, тем самым, только от $n$.

ДокАЗАТЕЛЬСтво. Согласно (27) имеем

$$
\left|g_{T}(x)\right| \leqslant 2(\pi|x|)^{-\nu} \sum_{k=1}^{\infty}\left|\frac{z_{k}}{2}\right|^{\nu+2}\left|H_{\nu}^{(1)}\left(z_{k}|x|\right)\right|, \quad|x|>0,
$$

где $\nu=(n-2) / 2, z_{k}=(1+i) \sqrt{k \pi / T}$. Подставим численные значения $z_{k}$ и воспользуемся оценкой Вебера (34). После элементарных подсчетов получим

$$
\left|g_{T}(x)\right| \leqslant C_{\nu} T^{-(2 \nu+3) / 4}|x|^{-(\nu+0.5)} \sum_{k=1}^{\infty} k^{(2 \nu+3) / 4} \exp \left(-\sqrt{\frac{k \pi}{T}}|x|\right)
$$


при $|x| \geqslant \sqrt{T /(2 \pi)}$ с константой $C_{\nu}>0$, зависяшей только от $\nu$. Ряд $\sum_{k=1}^{\infty}$, входящий в (46), есть ряд вида (41), где $\beta=(2 \nu+3) / 4=(n+1) / 4>0$ и $\rho=\sqrt{\pi / T}|x|$. Оценивая сумму ряда по принципу (43), заключаем, что

$$
\left|g_{T}(x)\right| \leqslant C|x|^{-(\nu+0.5)} \exp \left(-\sqrt{\frac{\pi}{T}}|x|\right), \quad|x| \geqslant r
$$

с константами $C>0$ и $r>0$, зависящими только от $\nu$ и $T$. Функция $g_{T}(x)$ непрерывна в $\mathbb{R}^{n}$. Неравенство (47) можно распространить на все $\mathbb{R}^{n} \backslash\{0\}$ при необходимом увеличении $C$. Так как $\nu+0.5=(n-1) / 2$, получили оценку $(44)$. Оценка (45) - очевидное следствие (44). Из определения (10) легко вьводится, что $g_{T}(x)=T^{-n / 2} g_{1}\left(T^{-1 / 2} x\right)$. Отсюда находятся значения $C_{T, n}=T^{-(n+1) / 4} C_{1, n}$ и $D_{T, n}=T^{-n / 2} D_{1, n}$. Лемма доказана.

В последующем доказательстве основной теоремы 3 используется именно лемма 6, точнее ее вариант с оценкой (45). Но в более специальных исследованиях может понадобиться дополнительная информация о поведении функции $g_{T}(x)$ на бесконечности. На этот случай отметим, что $k$-е слагаемое ряда (27) при $|x| \rightarrow \infty$ выражается приближенной формулой

$$
\begin{aligned}
-2(\pi|x|)^{-\nu} \operatorname{Im}\left(\left(\frac{z_{k}}{2}\right)^{\nu+2} H_{\nu}^{(1)}\left(z_{k}|x|\right)\right) \\
=-(2 \pi)^{-(n-3) / 4}\left(\frac{k}{T}\right)^{(n+1) / 4}|x|^{-(n-1) / 2} \exp \left(-\sqrt{\frac{k \pi}{T}}|x|\right) \\
\times\left[\sin \left(\sqrt{\frac{k \pi}{T}}|x|-\frac{\pi}{8}(n-3)\right)+O\left(|x|^{-1}\right)\right]
\end{aligned}
$$

с символом $O\left(|x|^{-1}\right)$, равномерньм по $k$. Формула (48) получается непосредственно при подстановке в левую часть $z_{k}=(1+i) \sqrt{k \pi / T}=e^{\pi i / 4} \sqrt{2 k \pi / T}$, $\nu=(n-2) / 2$ и использовании известной асимптотики функции Ханкеля:

$$
H_{\nu}^{(1)}(z)=\sqrt{\frac{2}{\pi z}} \exp \left(i z-\frac{\pi i}{4}(2 \nu+1)\right)\left[1+O\left(|z|^{-1}\right)\right], \quad|z| \rightarrow \infty,
$$

на луче $\arg z=\pi / 4$. Соответствующие вычисления элементарны, и мы их не приводим. При $\nu=-1 / 2$ и $\nu=1 / 2$ функции Ханкеля выглядят особенно просто приближенная формула (49) заменяется точным равенством (без $\left.O\left(|z|^{-1}\right)\right)$. Поэтому в формуле (48) при $n=1$ и $n=3$ символ $O\left(|x|^{-1}\right)$ можно опустить ${ }^{7}$.

При $|x| \rightarrow \infty$ все функции (48) являются осциллирующими с экспоненциально убывающими амплитудами. С помощью асимптотических разложений типа (41) можно показать, что при больших $|x|$ первое слагаемое ряда (27) является “доминируюшим" по отношению к остатку. Точнее, при $|x| \rightarrow \infty$ ряд (27) дает асимптотическое разложение в смысле Эрдейи (но не Пуанкаре; см. [16; гл. I, § 2]) для $g_{T}(x)$ по асимптотической последовательности

$$
\left\{|x|^{-(n-1) / 2} \exp \left(-\sqrt{\frac{k \pi}{T}}|x|\right)\right\}_{k=1}^{\infty} .
$$

\footnotetext{
${ }^{7}$ Случаи $n=1$ и $n=3$ наиболее интересны для приложений.
} 
Отсюда, в частности, следует, что сама функция $g_{T}(x)$ осциллирует (меняет знак) при $|x| \rightarrow \infty$.

Действительно, в разложении (27) отбросим первое слагаемое (с $k=1)$ и оценим оставшееся так же, как в доказательстве леммы 6. Получим выражение типа (46), где $\sum$ начинается с $k=2$. Рассмотрим эту сумму, как 1-й остаток ряда (41) с $\beta=(2 \nu+3) / 4=(n+1) / 4>0$ и $\rho=\sqrt{\pi / T}|x|$. Воспользуемся оценкой (42) для $m=2$. Аналогично (47) заключаем, что

$$
\left|2(\pi|x|)^{-\nu} \sum_{k=2}^{\infty} \operatorname{Im}\left(\left(\frac{z_{k}}{2}\right)^{\nu+2} H_{\nu}^{(1)}\left(z_{k}|x|\right)\right)\right| \leqslant C|x|^{-(\nu+0.5)} \exp \left(-\sqrt{\frac{2 \pi}{T}}|x|\right)
$$

при достаточно больших $|x|$ с подходящей константой $C>0$. После вынесения множителя $|x|^{-(\nu+0.5)}=|x|^{-(n-1) / 2}$ имеем величину $O(\exp (-\sqrt{2 \pi / T}|x|))$ и, тем самьп, $O\left(|x|^{-1} \exp (-\sqrt{\pi / T}|x|)\right)$. Совмешая это с приближенной формулой (48), взятой для первого слагаемого $(c k=1)$, получаем формулу для суммы:

$$
\begin{aligned}
g_{T}(x)= & -(2 \pi)^{-(n-3) / 4} T^{-(n+1) / 4}|x|^{-(n-1) / 2} \exp \left(-\sqrt{\frac{\pi}{T}}|x|\right) \\
& \times\left[\sin \left(\sqrt{\frac{\pi}{T}}|x|-\frac{\pi}{8}(n-3)\right)+O\left(|x|^{-1}\right)\right], \quad|x| \rightarrow \infty .
\end{aligned}
$$

При $n=1$ и $n=3$ слагаемое $O\left(|x|^{-1}\right)$ в (50) можно заменить на $O(\exp (-\gamma|x|))$ с $\gamma \equiv(\sqrt{2}-1) \sqrt{\pi / T}$. Асимптотическая формула $(50)$ и показывает, что функция $g_{T}(x)$ осциллирует при $|x| \rightarrow \infty$.

Кроме того, из (50) следует, что оценки сверху для $g_{T}(x)$, найденные в лемме 6 , являются окончательными. Напомним, что уже согласно лемме 2 оценка (45) точна в экспоненциальных классах $\mathscr{E}_{\sigma}\left(\mathbb{R}^{n}\right)$. Теперь стало ясно, что “тонкая” оценка (44) будет "абсолютно неулучшаемой”.

ЛЕмма 7. Функиию $|x|^{-(n-1) / 2} \exp (-\sqrt{\pi / T}|x|)$ в оценке (44) нельзя заменить никакой функиией $h(|x|)$ вида $о\left(|x|^{-(n-1) / 2} \exp (-\sqrt{\pi / T}|x|)\right)$ при $|x| \rightarrow \infty$.

ДокАЗАТЕЛьСтво. См. (50).

Функция $g_{T}(x)$ вообще весьма интересна. В ее исходном определении, в $\S 2$, использовалась функция $Q(s) \equiv s(\exp (s)-1)^{-1}$ - производящая функция чисел Бернулли. Поэтому в других представлениях для $g_{T}(x)$ появляются такие классические объекты анализа, как числа Бернулли, Һ-функция Римана и т.д. Например из $(29)$ выводится разложение $g_{T}(x)$ в сходящийся степенной ряд:

$$
g_{T}(x)=(4 \pi T)^{-n / 2} \sum_{k=0}^{\infty} \frac{1}{k !}\left(\frac{n}{2}+k\right) \zeta\left(\frac{n}{2}+k+1\right)\left(-\frac{|x|^{2}}{4 T}\right)^{k}, \quad x \in \mathbb{R}^{n}
$$

напоминаюший своими крайними частями фундаментальное решение уравнения теплопроводности. Для получения (51) надо подставить в (29) стандартное разложение в степенной ряд для функции Бесселя $J_{(n-2) / 2}(r|x|)$, провести элементарные преобразования интегралов (с заменой $T r^{2}$ на $s$ ) и воспользоваться тождеством

$$
\int_{0}^{\infty} \frac{s^{\alpha-1}}{e^{s}-1} d s=\Gamma(\alpha) \zeta(\alpha), \quad \alpha>1
$$


Несложные подсчеты дадут (51). Отсюда между прочим следует, что

$$
g_{T}(0)=(4 \pi T)^{-n / 2} \frac{n}{2} \cdot \zeta\left(\frac{n}{2}+1\right) .
$$

Например при $n=2$ имеем $g_{T}(0)=(4 \pi T)^{-1}\left(\pi^{2} / 6\right)=\pi /(24 T)$.

Формула (51) удобна для описания $g_{T}(x)$ вблизи нуля так же, как формула $(50)-$ на бесконечности.

\section{§ 8. Доказательство основной теоремы 3}

Первое утверждение теоремы 3 о единственности решения задачи (1), (2) следует из результатов работы [7] (см. также [6]).

Второе утверждение о необходимости условий $\varphi \in \mathscr{O}\left(\mathbb{R}^{n}\right) \cap \mathscr{E}_{\sigma}\left(\mathbb{R}^{n}\right)$ и $\Delta^{*} \varphi \in$ $\mathscr{E}_{\sigma}\left(\mathbb{R}^{n}\right)$ фактически уже доказано. Действительно, пусть $u(x, t)$ - решение задачи $(1),(2)$, принадлежащее $\mathscr{E}_{\sigma}\left(\mathbb{R}^{n} \times[0, T]\right)$. Согласно лемме 4 должно выполняться соотношение $\varphi \in \mathscr{O}\left(\mathbb{R}^{n}\right)$, причем очевидно, что функции $\varphi(x)$ и $\Delta^{*} \varphi(x)=$ $T^{-1}(u(x, T)-u(x, 0))$ принадлежат $\mathscr{E}_{\sigma}\left(\mathbb{R}^{n}\right)$.

Докажем остальные утверждения теоремы. Зафиксируем число $\sigma<\sqrt{\pi / T}$ и функцию $\varphi \in \mathscr{O}\left(\mathbb{R}^{n}\right) \cap \mathscr{E}_{\sigma}\left(\mathbb{R}^{n}\right)$ такую, что $\Delta^{*} \varphi \in \mathscr{E}_{\sigma}\left(\mathbb{R}^{n}\right)$. Пусть $\varphi(x)$ и $\Delta^{*} \varphi(x)$ удовлетворяют неравенствам $(22)$ с константой $M>0$. Покажем, что формулы (4), (21) действительно определяют решение $u(x, t)$ задачи $(1),(2)$, подпадающее под описание из пп. 4)-6) теоремы 3 в зависимости от значения $\sigma$. Поскольку размерность $n$, число $T>0$ и показатель $\sigma$ считаются заданными, мы не будем указывать на зависимость от $n, T, \sigma$ различных констант, возникающих в оценках. Однако будем следить за константой $M$, чтобы получить нужные оценки устойчивости.

Всюду в параграфе для краткости полагаем $\sigma_{0} \equiv \sqrt{\pi / T}$. Таким образом, $\sigma<\sigma_{0}$. Согласно лемме 6 для функции $g_{T}(x)$ можно указать число $D_{0}>0$ $\left(D_{0} \equiv D_{T, n}\right.$ в лемме 6) такое, что $\left|g_{T}(x)\right| \leqslant D_{0} \exp \left(-\sigma_{0}|x|\right)$ для $x \in \mathbb{R}^{n}$. Другими словами, функция $g_{T}(x)$ принадлежит классу $\mathscr{E}_{-\sigma_{0}}\left(\mathbb{R}^{n}\right)$.

Обратимся к формуле $(21)$ для начального состояния $u_{0}(x)$. По условию второе слагаемое $T \Delta^{*} \varphi(x)$ непрерывно в $\mathbb{R}^{n}$ и принадлежит классу $\mathscr{E}_{\sigma}\left(\mathbb{R}^{n}\right)$. Рассмотрим первое слагаемое, т.е. интеграл

$$
\chi(x) \equiv \int_{\mathbb{R}^{n}} g_{T}(x-y) \varphi(y) d y .
$$

Для подынтегральной функции справедлива оценка

$$
\left|g_{T}(x-y) \varphi(y)\right| \leqslant D_{0} \exp \left(-\sigma_{0}|x-y|\right) M \exp (\sigma|y|)
$$

Если $|x| \leqslant r$, то $\left|g_{T}(x-y) \varphi(y)\right| \leqslant M D_{0} \exp \left(\sigma_{0} r\right) \exp \left(-\left(\sigma_{0}-\sigma\right)|y|\right)$ при $y \in \mathbb{R}^{n}$. Поэтому интеграл (52) сходится при всех $x \in \mathbb{R}^{n}$ равномерно в любом шаре $|x| \leqslant r$ и представляет собой непрерывную функцию переменной $x \in \mathbb{R}^{n}$. Поведение интеграла при $|x| \rightarrow \infty$ зависит от того, в какой промежуток попадает исходное значение $\sigma$. 
Пусть $|\sigma|<\sigma_{0}$. Тогда

$$
\begin{aligned}
|\chi(x)| & \leqslant M D_{0} \int_{\mathbb{R}^{n}} e^{-\sigma_{0}|x-y|} e^{\sigma|y|} d y \\
& \leqslant M D_{0} e^{\sigma|x|} \int_{\mathbb{R}^{n}} e^{-\left(\sigma_{0}-|\sigma|\right)|y-x|} d y=M D_{1} e^{\sigma|x|}, \\
x & \in \mathbb{R}^{n}, \quad D_{1} \equiv D_{0} \int_{\mathbb{R}^{n}} e^{-\left(\sigma_{0}-|\sigma|\right)|y|} d y<\infty
\end{aligned}
$$

т.е. интеграл (52) принадлежит классу $\mathscr{E}_{\sigma}\left(\mathbb{R}^{n}\right)$. Следовательно, функция $u_{0}(x)$, заданная формулой $(21)$, непрерывна в $\mathbb{R}^{n}$, принадлежит классу $\mathscr{E}_{\sigma}\left(\mathbb{R}^{n}\right)$ и удовлетворяет оценке $\left|u_{0}(x)\right| \leqslant M\left(D_{1}+T\right) \exp (\sigma|x|)$ всюду в $\mathbb{R}^{n}$.

Определим для данного начального состояния $u_{0}(x)$ функцию $u(x, t)$ по формуле Пуассона (4). Тогда $u(x, t)$ служит решением уравнения теплопроводности (1) и по лемме 1 принадлежит $\mathscr{E}_{\sigma}\left(\mathbb{R}^{n} \times[0, T]\right)$. Справедлива оценка

$$
|u(x, t)| \leqslant M\left(D_{1}+T\right) M^{*} \exp (\sigma|x|), \quad x \in \mathbb{R}^{n}, \quad 0 \leqslant t \leqslant T
$$

где $M^{*}=2^{n} \exp \left(n \sigma^{2} T\right)$ как в доказательстве ${ }^{8}$ леммы 1 . Число $N \equiv\left(D_{1}+T\right) M^{*}$ зависит лишш от $n, T$ и $\sigma$. Получили оценку устойчивости (23).

Пусть теперь $\sigma<-\sigma_{0}$. Тогда $\sigma+\sigma_{0}<0$ и

$$
\begin{aligned}
|\chi(x)| & \leqslant M D_{0} \int_{\mathbb{R}^{n}} e^{-\sigma_{0}|x-y|} e^{\sigma|y|} d y \leqslant M D_{0} e^{-\sigma_{0}|x|} \int_{\mathbb{R}^{n}} e^{\left(\sigma_{0}+\sigma\right)|y|} d y \\
& =M D_{2} e^{-\sigma_{0}|x|}, \quad x \in \mathbb{R}^{n}, \quad D_{2} \equiv D_{0} \int_{\mathbb{R}^{n}} e^{\left(\sigma_{0}+\sigma\right)|y|} d y<\infty
\end{aligned}
$$

т.е. интеграл $(52)$ принадлежит классу $\mathscr{E}_{-\sigma_{0}}\left(\mathbb{R}^{n}\right)$. Следовательно, функция $u_{0}(x)$, заданная формулой $(21)$, непрерывна в $\mathbb{R}^{n}$, принадлежит $\mathscr{E}_{-\sigma_{0}}\left(\mathbb{R}^{n}\right)$ и удовлетворяет оценке $\left|u_{0}(x)\right| \leqslant M\left(D_{2}+T\right) \exp \left(-\sigma_{0}|x|\right)$ для $x \in \mathbb{R}^{n}$. Снова определим функцию $u(x, t)$ по формуле Пуассона $(4)$. Тогда $u(x, t)$ служит решением уравнения теплопроводности $(1)$, принадлежит $\mathscr{E}_{-\sigma_{0}}\left(\mathbb{R}^{n} \times[0, T]\right)$ и

$$
|u(x, t)| \leqslant M\left(D_{2}+T\right) M^{*} \exp \left(-\sigma_{0}|x|\right), \quad x \in \mathbb{R}^{n}, \quad 0 \leqslant t \leqslant T,
$$

где $M^{*}=2^{n} \exp \left(n \sigma_{0}^{2} T\right)=2^{n} \exp (n \pi)$. Получили оценку устойчивости $(24)$ с константой $N \equiv\left(D_{2}+T\right) M^{*}$, зависящей лишь от $n, T$ и $\sigma$.

Пусть, наконец, $\sigma=-\sigma_{0}$. Перейдем к чуть большему числу $\sigma_{1}$, считая, конечно, что $\left|\sigma_{1}\right|<\sigma_{0}$. Ясно, что $\varphi \in \mathscr{O}\left(\mathbb{R}^{n}\right) \cap \mathscr{E}_{\sigma_{1}}\left(\mathbb{R}^{n}\right)$ и $\Delta^{*} \varphi \in \mathscr{E}_{\sigma_{1}}\left(\mathbb{R}^{n}\right)$, причем $|\varphi(x)| \leqslant M \exp \left(\sigma_{1}|x|\right)$ и $\left|\Delta^{*} \varphi(x)\right| \leqslant M \exp \left(\sigma_{1}|x|\right)$ с той же константой $M>0$, что и в (22). На основании доказанного выше, функция $u(x, t)$, определенная формулами (4), (21), служит решением уравнения теплопроводности (1), принадлежит

\footnotetext{
${ }^{8}$ Указанное там значение $M^{*}$ несколько завьпшено. Более точное выражение получается через интеграл ошибок: $M^{*}=(1+\operatorname{erf}(|\sigma| \sqrt{T}))^{n} \exp \left(n \sigma^{2} T\right)$. В частности, при $\sigma=0$ можно считать $M^{*}=1$, а не $M^{*}=2^{n}$.
} 
классу $\mathscr{E}_{\sigma_{1}}\left(\mathbb{R}^{n} \times[0, T]\right)$ и удовлетворяет оценке устойчивости $(26)$ с константой $N>0$, зависяшей от $n, T, \sigma_{1}$. Ввиду произвольности выбранного $\sigma_{1}$ получаем включение (25).

Итак, во всех трех случаях формулы (4), (21) корректно определяют решение $u(x, t)$ уравнения теплопроводности (1), подпадаюшее под описание из пп. 4)-6) теоремы 3 в зависимости от значения $\sigma$. Осталось показать, что при подстановке $u(x, t)$ в условие $(2)$ получается исходная функция $\varphi(x)$. Собственно в этом и состоит основная часть доказательства.

Для сокращения записи обозначим через

$$
\mu_{t}(x) \equiv(4 \pi t)^{-n / 2} \exp \left(-\frac{|x|^{2}}{4 t}\right), \quad x \in \mathbb{R}^{n}, \quad t>0,
$$

фундаментальное решение уравнения теплопроводности. По формуле (4) функция $u(x, t)$ при $t>0$ есть свертка $\mu_{t}(x)$ с начальньм состоянием $u_{0}(x)$. Используя (21), разобьем $u(x, t)$ на два слагаемых:

$$
u(x, t)=u_{1}(x, t)-T u_{2}(x, t) .
$$

Здесь $u_{1}(x, t)$ - решение уравнения теплопроводности, полученное по формуле Пуассона для начального состояния $\chi(x)$ из $(52)$, т.е.

$$
\begin{aligned}
\left.u_{1}(x, t)\right|_{0<t \leqslant T} & =\int_{\mathbb{R}^{n}} \mu_{t}(x-\xi) d \xi \int_{\mathbb{R}^{n}} g_{T}(\xi-y) \varphi(y) d y, \\
u_{1}(x, 0) & =\int_{\mathbb{R}^{n}} g_{T}(x-y) \varphi(y) d y .
\end{aligned}
$$

Второе решение $u_{2}(x, t)$ отвечает начальному условию $\Delta^{*} \varphi(x)$, т.е.

$$
\left.u_{2}(x, t)\right|_{0<t \leqslant T}=\int_{\mathbb{R}^{n}} \mu_{t}(x-y) \Delta^{*} \varphi(y) d y, \quad u_{2}(x, 0)=\Delta^{*} \varphi(x) .
$$

Рассмотрим эти решения отдельно.

Начнем с $u_{1}(x, t)$. Для функции $\mu_{t}(x-\xi) g_{T}(\xi-y) \varphi(y)$ при фиксированном $(x, t) \in \mathbb{R}^{n} \times(0, T]$ справедлива оценка

$$
\begin{aligned}
\left|\mu_{t}(x-\xi) g_{T}(\xi-y) \varphi(y)\right| & \leqslant(4 \pi t)^{-n / 2} e^{-|x-\xi|^{2} /(4 t)} D_{0} e^{-\sigma_{0}|\xi-y|} M e^{\sigma|y|} \\
& \leqslant C e^{-\alpha|\xi|^{2}+\beta|\xi|} e^{-\left(\sigma_{0}-\sigma\right)|y|}, \quad(\xi, y) \in \mathbb{R}^{n} \times \mathbb{R}^{n}
\end{aligned}
$$

где положительные константы $C, \alpha, \beta$ зависят, вообще говоря, от $(x, t)$. Подынтегральная функция в повторном интеграле (55) оказывается суммируемой на $\mathbb{R}^{n} \times \mathbb{R}^{n}$ по мере $d \xi d y$, и, значит, в этом интеграле можно переменить порядок интегрирования. Получим

$$
\begin{aligned}
\left.u_{1}(x, t)\right|_{0<t \leqslant T} & =\int_{\mathbb{R}^{n}} \varphi(y) d y \int_{\mathbb{R}^{n}} \mu_{t}(x-\xi) g_{T}(\xi-y) d \xi \\
& =\int_{\mathbb{R}^{n}} \varphi(y) d y \int_{\mathbb{R}^{n}} \mu_{t}(x-y-\xi) g_{T}(\xi) d \xi
\end{aligned}
$$


Определим новую функцию:

$$
\left.v(x, t)\right|_{0<t \leqslant T}=\int_{\mathbb{R}^{n}} \mu_{t}(x-\xi) g_{T}(\xi) d \xi, \quad v(x, 0)=g_{T}(x),
$$

т.е. решение уравнения теплопроводности с начальньм состоянием $g_{T}(x)$. Учитывая (56) и (58), запишем функцию $u_{1}(x, t)$ в виде

$$
u_{1}(x, t)=\int_{\mathbb{R}^{n}} v(x-y, t) \varphi(y) d y, \quad 0 \leqslant t \leqslant T .
$$

Особая роль специального решения (59) ясна из следующей леммы.

ЛЕмМа 8. Функиия $v(x, t)$, определенная формулой (59), есть решение уравнения теплопроводности (1) из класса $C^{2,1}\left(\mathbb{R}^{n} \times(0, T]\right) \cap C\left(\mathbb{R}^{n} \times[0, T]\right)$, удовлетворяющее оценке

$$
|v(x, t)| \leqslant C_{0} \exp \left(-\sigma_{0}|x|\right), \quad x \in \mathbb{R}^{n}, \quad 0 \leqslant t \leqslant T, \quad \sigma_{0} \equiv \sqrt{\frac{\pi}{T}}
$$

с некоторой константой $C_{0}>0$. Кроме того,

$$
\frac{1}{T} \int_{0}^{T} v(x, t) d t=\mu_{T}(x), \quad x \in \mathbb{R}^{n}
$$

ЗАмечАниЕ 2. Равенство (62) окончательно раскрывает связь функции $g_{T}(x)$ с нелокальной задачей $(1),(2)$. Оказывается, решение уравнения теплопроводности (1), отвечающее начальному состоянию $g_{T}(x)$, при подстановке в $(2)$ дает фундаментальное решение $\mu_{T}(x)$ в момент времени $t=T$. Используя стандартное обозначение для свертки по переменной $x \in \mathbb{R}^{n}$, равенство (62) можно записать в следующем наглядном виде:

$$
\frac{1}{T} \int_{0}^{T} \mu_{t} * g_{T} d t=\mu_{T}
$$

Отметим соотношение $\mu_{T} * g_{T}=g_{T}+T \Delta \mu_{T}$, которое получается из (63) применением леммы 4 (или легко может быть проверено с помощью преобразования Фурье).

ДОКАЗАТЕЛЬСТво ЛЕМмы 8. Поскольку $\left|g_{T}(x)\right| \leqslant D_{0} \exp \left(-\sigma_{0}|x|\right)$ при $x \in \mathbb{R}^{n}$, то первая часть леммы, включая оценку (61), немедленно следует из формулы Пуассона (59) и леммы 1. Докажем равенство (62). Рассмотрим преобразование Фуpьe

$$
V(\xi, t)=\int_{\mathbb{R}^{n}} v(x, t) \exp (-i \xi x) d x, \quad \xi \in \mathbb{R}^{n}, \quad 0 \leqslant t \leqslant T
$$


Напомним, что все функции, входящие в (59), принадлежат пространству Шварца $S\left(\mathbb{R}^{n}\right)$ (см. $\left.\S 2\right)$ и, значит, манипуляпии с преобразованием Фурье законны. Учитьвая, что преобразование от $\mu_{t}(x)$ есть $\exp \left(-|\xi|^{2} t\right)$, а преобразование от $g_{T}(x)$ есть функция $G_{T}(\xi)$ из $(9)$, получаем по теореме о свертке:

$$
V(\xi, t)=\exp \left(-|\xi|^{2} t\right) G_{T}(\xi)=\exp \left(-|\xi|^{2} t\right) \frac{T|\xi|^{2}}{\exp \left(T|\xi|^{2}\right)-1},
$$

причем это равенство справедливо также при $t=0$ (см. (59)). Но тогда

$$
\frac{1}{T} \int_{0}^{T} V(\xi, t) d t=\frac{|\xi|^{2}}{\exp \left(T|\xi|^{2}\right)-1} \int_{0}^{T} \exp \left(-|\xi|^{2} t\right) d t=\exp \left(-T|\xi|^{2}\right) .
$$

С помощью оценки (61) легко обосновать законность внесения интеграла по $t \in$ $[0, T]$ под знак интеграла (64). Таким образом, преобразование Фурье от непрерывной, экспоненциально убывающей функции

$$
\frac{1}{T} \int_{0}^{T} v(x, t) d t, \quad x \in \mathbb{R}^{n}
$$

совпадает с $\exp \left(-T|\xi|^{2}\right)$, т.е. с преобразованием от $\mu_{T}(x)$. Отсюда следует равенство (62). Лемма доказана.

Вернемся к представлению (60). Из той же оценки (61) вытекает, что

$|v(x-y, t) \varphi(y)| \leqslant C_{0} \exp \left(-\sigma_{0}|x-y|\right) M \exp (\sigma|y|) \leqslant C_{0} M e^{\sigma_{0}|x|} \exp \left(-\left(\sigma_{0}-\sigma\right)|y|\right)$,

т.е. при фиксированном $x \in \mathbb{R}^{n}$ функция $v(x-y, t) \varphi(y)$ является суммируемой на $\mathbb{R}^{n} \times[0, T]$ по $d y d t$. Поэтому

$$
\frac{1}{T} \int_{0}^{T} u_{1}(x, t) d t=\int_{\mathbb{R}^{n}}\left(\frac{1}{T} \int_{0}^{T} v(x-y, t) d t\right) \varphi(y) d y=\int_{\mathbb{R}^{n}} \mu_{T}(x-y) \varphi(y) d y .
$$

Интеграл вида (2) от первого слагаемого в (54) вычислен.

Перейдем к $u_{2}(x, t)$. По определению $(57)$ функция $u_{2}(x, t)$ есть решение уравнения теплопроводности, отвечаюшее начальному состоянию $\Delta^{*} \varphi(x)$. В силу леммы 4 при любом $\tau>0$ имеем

$$
\Delta^{*}\left(\int_{0}^{\tau} u_{2}(x, t) d t\right)=u_{2}(x, \tau)-\Delta^{*} \varphi(x), \quad x \in \mathbb{R}^{n},
$$

откуда

$$
\Delta^{*}\left(\varphi(x)+\int_{0}^{\tau} u_{2}(x, t) d t\right)=u_{2}(x, \tau), \quad x \in \mathbb{R}^{n} .
$$

Определим функцию

$$
w(x, \tau)=\varphi(x)+\int_{0}^{\tau} u_{2}(x, t) d t, \quad x \in \mathbb{R}^{n}, \quad 0 \leqslant \tau \leqslant T .
$$


Ясно, что функции $w(x, \tau)$ и $\partial w(x, \tau) / \partial \tau=u_{2}(x, \tau)$ непрерывны в $\mathbb{R}^{n} \times[0, T]$. В силу (66) при фиксированном $\tau_{0} \in(0, T]$ функция $\Delta^{*} w\left(x, \tau_{0}\right)$ равна $u_{2}\left(x, \tau_{0}\right)$ и, следовательно, принадлежит $C^{2}\left(\mathbb{R}^{n}\right)$ (и даже $\left.C^{\infty}\left(\mathbb{R}^{n}\right)\right)$. По свойствам 4 и 1 оператора $\Delta^{*}$ (см. $\left.\S 5\right)$ функция $w\left(x, \tau_{0}\right)$ принадлежит $C^{3}\left(\mathbb{R}^{n}\right)$ (точнее $C^{\infty}\left(\mathbb{R}^{n}\right)$ ) и $\Delta w\left(x, \tau_{0}\right)=\Delta^{*} w\left(x, \tau_{0}\right)$. В любом случае заведомо можно утверждать, что $w \in$ $C^{2,1}\left(\mathbb{R}^{n} \times(0, T]\right) \cap C\left(\mathbb{R}^{n} \times[0, T]\right)$ и

$$
\frac{\partial w(x, \tau)}{\partial \tau}=u_{2}(x, \tau)=\Delta^{*} w(x, \tau)=\Delta w(x, \tau), \quad x \in \mathbb{R}^{n}, \quad 0<\tau \leqslant T .
$$

Таким образом, функция $w(x, \tau)$ есть классическое решение уравнения теплопроводности, причем $w(x, 0)=\varphi(x)($ см. $(67))$. По условию $\varphi \in \mathscr{E}_{\sigma}\left(\mathbb{R}^{n}\right)$ и $\Delta^{*} \varphi \in$ $\mathscr{E}_{\sigma}\left(\mathbb{R}^{n}\right)$. По лемме $1 u_{2} \in \mathscr{E}_{\sigma}\left(\mathbb{R}^{n} \times[0, T]\right)$ как функция, заданная формулой Пуассона $(57)$ с начальньм состоянием $\Delta^{*} \varphi(x)$. Но тогда и $w \in \mathscr{E}_{\sigma}\left(\mathbb{R}^{n} \times[0, T]\right)$ согласно определению (67). При заданном начальном состоянии решение уравнения теплопроводности в классе $\mathscr{E}_{\sigma}\left(\mathbb{R}^{n} \times[0, T]\right)$ единственно; оно вычисляется по формуле Пуассона:

$$
\left.w(x, \tau)\right|_{0<\tau \leqslant T}=\int_{\mathbb{R}^{n}} \mu_{\tau}(x-y) \varphi(y) d y, \quad w(x, 0)=\varphi(x) .
$$

Подставив в (67) значение $\tau=T$, имеем

$$
\int_{0}^{T} u_{2}(x, t) d t=w(x, T)-\varphi(x)=\int_{\mathbb{R}^{n}} \mu_{T}(x-y) \varphi(y) d y-\varphi(x) .
$$

Интеграл от второго слагаемого в (54) вычислен.

Используя найденные выражения (65) и (68), получаем

$$
\begin{aligned}
& \frac{1}{T} \int_{0}^{T} u(x, t) d t=\frac{1}{T} \int_{0}^{T} u_{1}(x, t) d t-\int_{0}^{T} u_{2}(x, t) d t \\
& \quad=\int_{\mathbb{R}^{n}} \mu_{T}(x-y) \varphi(y) d y-\left(\int_{\mathbb{R}^{n}} \mu_{T}(x-y) \varphi(y) d y-\varphi(x)\right)=\varphi(x),
\end{aligned}
$$

т.е. функция $u(x, t)$, определенная по формулам $(4),(21)$, обрашает соотношение (2) в верное тождество. Итак, $u(x, t)$ является требуемым решением задачи $(1),(2)$. Это завершает доказательство утверждений 3)-6) теоремы 3. Теорема 3 полностью доказана.

Напомним, что частные теоремы 1, 2 немедленно следуют из теоремы 3 и потому не нуждаются в отдельных доказательствах.

\section{§9. Комментарий к теореме 3}

Снова обозначим $\sigma_{0} \equiv \sqrt{\pi / T}$. Полученные результаты указывают на наличие у задачи (1), (2) не только верхнего "критического" значения $\sigma=\sigma_{0}$, после которого теряется единственность, но и нижнего значения $\sigma=-\sigma_{0}$. 
При $|\sigma|<\sigma_{0}$ картина получается стереотипной: если $\varphi \in \mathscr{O}\left(\mathbb{R}^{n}\right) \cap \mathscr{E}_{\sigma}\left(\mathbb{R}^{n}\right)$ и $\Delta^{*} \varphi \in \mathscr{E}_{\sigma}\left(\mathbb{R}^{n}\right)$, то формулы (4), (21) определяют решение $u(x, t)$, принадлежащее $\mathscr{E}_{\sigma}\left(\mathbb{R}^{n} \times[0, T]\right)$. Здесь показатель роста для $u(x, t)$ не превосходит показателя для $\varphi(x)$ и $\Delta^{*} \varphi(x)$. Значения $\sigma \in\left(-\sigma_{0}, \sigma_{0}\right)$ являются "регулярньми" для задачи $(1),(2)$.

Это обстоятельство можно интерпретировать по-другому, по принципу "не от функции $\varphi(x)$ к решению $u(x, t)$ ", а наоборот, “от решения $u(x, t)$ к функции $\varphi(x)$ " или, что эквивалентно, "к функции $\psi(x)$ " из формулы (3). Зафиксируем регулярньй показатель $\sigma \in\left(-\sigma_{0}, \sigma_{0}\right)$. Пусть $u(x, t)$ - решение уравнения теплопроводности $(1)$, причем известно, что $u \in \mathscr{E}_{\sigma}\left(\mathbb{R}^{n} \times[0, T]\right)$, но $u \notin \mathscr{E}_{\sigma_{1}}\left(\mathbb{R}^{n} \times[0, T]\right)$ для любого $\sigma_{1}<\sigma$. Определим функцию $\psi(x)$ по формуле $(3)$. Очевидно, что $\psi \in \mathscr{E}_{\sigma}\left(\mathbb{R}^{n}\right)$. Но, кроме того, из теоремы 3 вытекает, что $\psi \notin \mathscr{E}_{\sigma_{1}}\left(\mathbb{R}^{n}\right)$ при любом $\sigma_{1}<\sigma$. Другими словами, регулярный показатель роста $\sigma$ не уменьшается при интегрировании по $t \in[0, T]$ решения $u(x, t)$.

При $\sigma<-\sigma_{0}$ ситуация меняется. Пусть $\varphi \in \mathscr{O}\left(\mathbb{R}^{n}\right) \cap \mathscr{E}_{\sigma}\left(\mathbb{R}^{n}\right)$ и $\Delta^{*} \varphi \in \mathscr{E}_{\sigma}\left(\mathbb{R}^{n}\right)$ с некоторьм $\sigma<-\sigma_{0}$. Из теоремы 3 следует, что формулы $(4),(21)$ определяют решение $u(x, t)$, принадлежашее $\mathscr{E}_{-\sigma_{0}}\left(\mathbb{R}^{n} \times[0, T]\right)$, но остается ли решение в $\mathscr{E}_{\sigma}\left(\mathbb{R}^{n} \times[0, T]\right)$ заранее не ясно. То, что переход в более широкий класс действительно возможен, видно на примере функции $v(x, t)$ из леммы 8. Рассмотрим эту функцию как решение $u \equiv v(x, t)$ задачи $(1),(2)$ с начальным состоянием $u_{0}(x) \equiv g_{T}(x)$ и функцией $\varphi(x) \equiv \mu_{T}(x)$. Здесь $\mu_{T}(x)$ - фундаментальное решение из формулы (53), взятое при $t=T$. Поэтому $\varphi(x)$ и $\Delta \varphi(x)$ принадлежат $\mathscr{E}_{\sigma}\left(\mathbb{R}^{n}\right)$ при любом $\sigma<-\sigma_{0}$. В то же время $u_{0}(x) \equiv g_{T}(x)$ не попадает в $\mathscr{E}_{\sigma}\left(\mathbb{R}^{n}\right)$ при $\sigma<-\sigma_{0}$, а принадлежит только $\mathscr{E}_{-\sigma_{0}}\left(\mathbb{R}^{n}\right)$ (см. леммы 2,6$)$. Соответственно, $u \notin \mathscr{E}_{\sigma}\left(\mathbb{R}^{n} \times[0, T]\right)$ при $\sigma<-\sigma_{0}$. Итак, сушествуют функции $u(x, t)$, для которых утверждение 5) в теореме 3 является неулучшаемьм.

Также неулучшаемым (по крайней мере, в экспоненциальных классах $\mathscr{E}_{\sigma}$ ) будет утверждение 6 ) теоремы 3 . Оказьвается, решение $u(x, t)$, полученное по формулам (4), (21) для $\varphi \in \mathscr{O}\left(\mathbb{R}^{n}\right) \cap \mathscr{E}_{-\sigma_{0}}\left(\mathbb{R}^{n}\right)$ и $\Delta^{*} \varphi \in \mathscr{E}_{-\sigma_{0}}\left(\mathbb{R}^{n}\right)$, может выходить из $\mathscr{E}_{-\sigma_{0}}\left(\mathbb{R}^{n} \times[0, T]\right)$, сохраняясь лишь в классе $(25)$. Этот эффект связан с появлением дополнительных степенных множителей типа $|x|^{\alpha}$ в асимптотике $|u(x, t)|$ при $|x| \rightarrow \infty$. Не проводя детальных исследований, ограничимся одним примером.

ПримеР. Пусть $n=1, \mathbb{R}^{n}=\mathbb{R}^{1}=\mathbb{R}$. Положим также $T=1$. Тогда $\sigma_{0} \equiv$ $\sqrt{\pi / T}=\sqrt{\pi}$. Функцию Грина из формулы (10) запишем в виде

$$
g_{1}(x)=\frac{1}{2 \pi} \int_{-\infty}^{\infty} \frac{s^{2}}{\exp \left(s^{2}\right)-1} \exp (\text { is } x) d s, \quad-\infty<x<\infty,
$$

с преобразованием Фурье $G_{1}(s)=s^{2}\left(\exp \left(s^{2}\right)-1\right)^{-1},-\infty<s<\infty$. (Мы обозначаем одномерную переменную через $s$ вместо прежней многомерной $\xi$.) Функция $g_{1}(x)$ является четной, в ее исследовании можно ограничиться $x>0$.

Воспользуемся разложением в ряд для $g_{1}(x)$, указанным в $\S 7$. Слагаемые ряда выражаются формулой (48), которая для $n=1$ будет точной без символа $O\left(|x|^{-1}\right)$. При $x>0$ получаем

$$
g_{1}(x)=-\sum_{k=1}^{\infty} \sqrt{2 k \pi} \exp (-\sqrt{k \pi} x) \sin \left(\sqrt{k \pi} x+\frac{\pi}{4}\right) .
$$


Ряд (70) можно почленно дифференцировать по $x$ любое число раз - возникающие ряды из производных равномерно сходятся на лучах $[a, \infty) \subset(0, \infty)$. В частности, имеем

$$
\begin{array}{ll}
g_{1}^{\prime}(x)=\sum_{k=1}^{\infty} 2 k \pi \exp (-\sqrt{k \pi} x) \sin (\sqrt{k \pi} x), & x>0 \\
g_{1}^{\prime \prime}(x)=\sum_{k=1}^{\infty}(2 k \pi)^{3 / 2} \exp (-\sqrt{k \pi} x) \cos \left(\sqrt{k \pi} x+\frac{\pi}{4}\right), & x>0
\end{array}
$$

и т.д. Используя элементарные оценки и лемму 5 , заключаем, что все производные $g_{1}^{(j)}(x)$, включая саму функцию $g_{1}(x)$, принадлежат $\mathscr{E}_{-\sigma_{0}}(\mathbb{R})$. С помошњю той же леммы 5 можно оценивать остатки в разложениях для производных. Например можно утверждать, что

$$
g_{1}^{\prime}(x)=2 \pi \exp (-\sqrt{\pi} x) \sin (\sqrt{\pi} x)+O(\exp (-\sqrt{2 \pi} x))
$$

при $x \rightarrow+\infty$.

Рассмотрим нелокальную задачу

$$
\frac{\partial u}{\partial t}=\frac{\partial^{2} u}{\partial x^{2}}, \quad \int_{0}^{1} u(x, t) d t=g_{1}(x)
$$

т.е. одномерную модель $(-\infty<x<\infty)$ для общей нелокальной задачи $(1),(2)$. Здесь $\varphi(x) \equiv g_{1}(x)$ и $\Delta \varphi(x) \equiv g_{1}^{\prime \prime}(x)$ принадлежат классу $\mathscr{E}_{-\sigma_{0}}(\mathbb{R})$. Поэтому задача (72) подпадает под утверждение 6) теоремы 3 . Определим начальное состояние $u_{0}(x)$ по формуле (11). Точнее, воспользуемся тем, что функция $\varphi(x) \equiv g_{1}(x)$ принадлежит пространству Шварца $S(\mathbb{R})$, и перейдем к эквивалентной формуле (12), согласно которой получим

$$
\begin{aligned}
u_{0}(x) & =\frac{1}{2 \pi} \int_{-\infty}^{\infty} P_{1}(s) G_{1}(s) e^{i s x} d s=\frac{1}{2 \pi} \int_{-\infty}^{\infty} \frac{s^{2} e^{s^{2}}}{e^{s^{2}}-1} \cdot \frac{s^{2}}{e^{s^{2}}-1} \cdot e^{i s x} d s \\
& =\frac{1}{2 \pi} \int_{-\infty}^{\infty} \frac{s^{4} e^{s^{2}} e^{i s x}}{\left(e^{s^{2}}-1\right)^{2}} d s
\end{aligned}
$$

Будем интегрировать по частям:

$$
\begin{aligned}
u_{0}(x) & =-\frac{1}{4 \pi} \int_{-\infty}^{\infty} s^{3} e^{i s x} d\left(\frac{1}{e^{s^{2}}-1}\right) \\
& =-\frac{1}{4 \pi} \frac{s^{3} e^{i s x}}{e^{s^{2}-1}}\left(\left.\right|_{-\infty} ^{-0}+\left.\right|_{+0} ^{+\infty}\right)+\frac{1}{4 \pi} \int_{-\infty}^{\infty} \frac{\left(3 s^{2}+s^{3} \cdot i x\right) e^{i s x}}{e^{s^{2}}-1} d s \\
& =0+\frac{3}{4 \pi} \int_{-\infty}^{\infty} \frac{s^{2} e^{i s x}}{e^{s^{2}}-1} d s+\frac{x}{4 \pi} \int_{-\infty}^{\infty} \frac{s^{2} \cdot i s e^{i s x}}{e^{s^{2}}-1} d s
\end{aligned}
$$


Вспоминая определение (69) функции $g_{1}(x)$, заключаем, что

$$
u_{0}(x)=\frac{3}{2} g_{1}(x)+\frac{1}{2} x g_{1}^{\prime}(x), \quad-\infty<x<\infty .
$$

Воспользуемся соотношением $(71)$ и тем, что $g_{1} \in \mathscr{E}_{-\sigma_{0}}(\mathbb{R})$ с $\sigma_{0} \equiv \sqrt{\pi}$. В итоге имеем асимптотику

$$
u_{0}(x)=\pi x \exp (-\sqrt{\pi} x) \sin (\sqrt{\pi} x)+O(\exp (-\sqrt{\pi} x))
$$

при $x \rightarrow+\infty$. Отсюда вытекает, что $u_{0} \notin \mathscr{E}_{-\sigma_{0}}(\mathbb{R})$. Но тогда и решение $u(x, t)$ задачи (72), полученное по формулам (4), (11), не попадает в $\mathscr{E}-\sigma_{0}(\mathbb{R} \times[0, T])$, хотя очевидно принадлежит соответствующему классу (25). Построение примера завершено.

Пример основан на появлении дополнительного степенного множителя $x$ в разложении $(73)$ перед $\exp (-\sqrt{\pi} x)$. Спрашивается, могут ли появиться $x^{2}, x^{3}$ и т.д.? В одномерном случае, по-видимому, нет, а в обшем многомерном случае стоит ожидать $|x|^{\alpha}$ с показателем $\alpha$, зависяшим от размерности $n$. Дальнейшее исследование лучше провести в рамках полного изучения разрешимости нелокальной задачи (1), (2) в экспоненциально-степенных классах функций с мажорантами $|x|^{a} \exp (\sigma|x|)$. Этот круг вопросов открыт и весьма интересен. Подготовительные соображения имеются в $\S 7$ (см. лемму 6 с оценкой (44), лемму 7 и приближенные формулы (48), (50)).

\section{§10. Специальные уточнения теоремы 3}

В формулировке теоремы 3 функции $\varphi(x)$ и $\Delta^{*} \varphi(x)$ были фактически равноправны - они фигурировали в схожих оценках (22) с единым показателем $\sigma$. Однако их “вклад” в основную формулу (21) очевидно различен: функция $\varphi(x)$ входит в оператор свертки с фиксированной функцией $g_{T}(x)$, а $\Delta^{*} \varphi(x)$ находится в отдельном слагаемом. Учитывая это различие, можно несколько дополнить теорему 3. Приведем следующие два результата.

TEOPEMA 5. Пусть $\varphi \in \mathscr{O}\left(\mathbb{R}^{n}\right) \cap \mathscr{E}_{\sigma_{1}}\left(\mathbb{R}^{n}\right) c \sigma_{1}<\sqrt{\pi / T}, a \Delta^{*} \varphi \in \mathscr{E}_{\sigma_{2}}\left(\mathbb{R}^{n}\right)$ с $\sigma_{2}<\infty$. Тогда задача (1), (2) разрешима - формуль (4), (21) определяют решение $u(x, t)$ задачи (1), (2). При $\sigma_{2} \geqslant \sqrt{\pi / T}$ это решение принадлежит $\mathscr{E}_{\sigma_{2}}\left(\mathbb{R}^{n} \times[0, T]\right)$, но является неединственным в данном классе (имеются другие решения, не связанные с формулами (4), (21)). При $\sigma_{2}<\sqrt{\pi / T}$ решение $u(x, t)$ подпадает под описание теоремы 3 со значением $\sigma=\max \left(\sigma_{1}, \sigma_{2}\right)$.

Tеорема 6. Пусть $\varphi \in \mathscr{O}\left(\mathbb{R}^{n}\right) \cap \mathscr{E}_{\sigma}\left(\mathbb{R}^{n}\right)$ с некоторицм $\sigma<-\sigma_{0} \equiv-\sqrt{\pi / T}$, a $\Delta^{*} \varphi \in \mathscr{E}_{-\sigma_{0}}\left(\mathbb{R}^{n}\right)$. Тогда формуль (4), (21) определяют решение $u(x, t)$ задачи (1), (2), принадлежсащее $\mathscr{E}_{-\sigma_{0}}\left(\mathbb{R}^{n} \times[0, T]\right)$.

Теорема 5 показьвает, что для применения формул (4), (21) существенно ограничение на рост $(\sigma<\sqrt{\pi / T})$ только для функции $\varphi(x)$. Например, по теореме 5 задача (1), (2) с функцией

$$
\varphi(x)=\sin \left(\exp \left(x_{1}+\cdots+x_{n}\right)\right), \quad x=\left(x_{1}, \ldots, x_{n}\right) \in \mathbb{R}^{n},
$$


будет разрешимой по формулам (4), (21) на любом промежутке $[0, T]$. Основная теорема 3 применима здесь лишь при $T<\pi /(4 n)$ из-за поведения $|\Delta \varphi(x)|$ при $|x| \rightarrow \infty$ : непосредственно проверяется, что $\Delta \varphi \in \mathscr{E}_{\sigma}\left(\mathbb{R}^{n}\right)$ для $\sigma=2 \sqrt{n}$ и данное значение $\sigma$ нельзя уменьшить.

Теорема 6 уточняет утверждение 6 ) теоремы 3 (см. также пример в $\S 9$ ). Оказывается, для перехода решения $u(x, t)$ в класс $(25)$ необходимо, чтобы именно $\varphi(x)$ принадлежала $\mathscr{E}_{-\sigma_{0}}\left(\mathbb{R}^{n}\right)$. Если $\Delta^{*} \varphi \in \mathscr{E}_{-\sigma_{0}}\left(\mathbb{R}^{n}\right)$, но $\varphi \in \mathscr{E}_{\sigma}\left(\mathbb{R}^{n}\right)$ с $\sigma<-\sigma_{0}$, то решение остается в $\mathscr{E}_{-\sigma_{0}}\left(\mathbb{R}^{n} \times[0, T]\right)$ и переход в более широкий класс $(25)$ не происходит.

Доказательство всех этих утверждений повторяет mutatis mutandis доказательство теоремы 3. Просматривая шаг за шагом рассуждения в $\S 8$, убеждаемся в справедливости теорем 5 и 6 . Неединственность решения (теорема 5 ) в классе $\mathscr{E}_{\sigma_{2}}\left(\mathbb{R}^{n} \times[0, T]\right)$ с $\sigma_{2} \geqslant \sqrt{\pi / T}$ следует из результатов работы [7]. Например, наряду с $u(x, t)$ решением той же задачи $(1),(2)$ будут функции

$$
u(x, t)+\exp \left(\sqrt{\frac{\pi}{T}} x_{k}\right) \cos \left(\sqrt{\frac{\pi}{T}} x_{k}+\frac{2 \pi}{T} t\right), \quad k=1, \ldots, n,
$$

где $x_{k}$ есть $k$-я координата точки $x$.

Авторы благодарны В. А. Ильину и А.И. Прилепко за обсуждения и поддержку этой работы.

\section{Список литературы}

1. Holmgren E. Sur les solutions quasianalytiques de l'équation de la chaleur // Ark. Mat. 1924. V. 18. № 9. P. 1-9.

2. Tychonoff $A$. Théorèmes d'unicitépour l'équation de la chaleur // Матем. сб. 1935. Т. 42. № 2. C. 199-216.

3. Täcklind S. Sur les classes quasianalytiques des solutions des équations aux dérivées partielles du type parabolique // Nova Acta Reg. Soc. Sci. Upsaliensis. Ser. IV. 1936. V. 10. №3. P. 1-57.

4. Гельфанд И. М., Шилов Г. Е. Обобщенные функции. Вып 3. Некоторые вопросы теории дифференциальных уравнений. М.: ГИФМЛ, 1958.

5. Тихонов И. В. Теоремы единственности в линейных нелокальных задачах для абстрактных дифференциальных уравнений // Изв. РАН. Сер. матем. 2003. Т. 67. № 2. С. 133-166.

6. Попов А.Ю., Тихонов И. В. Экспоненциальные классы единственности в задачах теплопроводности // Докл. РАН. 2003. Т. 389. № 4. С. 465-467.

7. Попов А. Ю., Тихонов И. В. Классы единственности в нелокальной по времени задаче для уравнения теплопроводности и комплексные собственные функции оператора Лапласа // Дифференц. уравнения. 2004. Т. 40. №3. С. 396-405.

8. Волевич Л.Р., Гиндикин С.Г. Обобщенные функции и уравнения в свертках. М.: Физматлит, 1994.

9. Михайлов В.П. Дифференциальные уравнения в частных производных. М.: Наука, 1983.

10. Привалов И. И. Субгармонические функции. М.: ГРТТЛ, 1937.

11. Гюнтер H. M. Теория потенциала и ее применение к основным задачам математической физики. М.: ГИТТЛ, 1953.

12. Бейтмен Г., Эрдейи А. Высшие трансцендентные функции. Т. 2. Функции Бесселя, функции параболического цилиндра, ортогональные многочлены. М.: Наука, 1974.

13. Абрамович М., Стиган И. (ред.) Справочник по специальным функциям с формулами, граффиками и математическими таблицами. М.: Наука, 1979. 
14. Прудников А.П., Брычков Ю. А., Маричев О. И. Интегралы и ряды. Дополнительные главы. М.: Наука, 1986.

15. Математическая энциклопедия. Т. 1. А-Г. М.: СЭ, 1977.

16. Ватсон Г. Н. Теория бесселевых функций. Часть первая. М.: ИЛ, 1949.

17. Федорюк М. В. Асимптотика: Интегралы и ряды. М.: Наука, 1987.

Московский государственнй университет

Поступила в редакцию им. М.В. Ломоносова,

14.10 .2004

Московский инженерно-ффизический институт 